\title{
A Molecular-line Study of the Interstellar Bullet Engine IRAS05506+2414
}

\author{
Raghvendra Sahai ${ }^{1}$ (1) ${ }_{1}$, Chin-Fei Lee ${ }^{2}$ (10, Carmen Sánchez Contreras ${ }^{3}$ (i), Nimesh Patel ${ }^{4}$, Mark R. Morris $^{5}$ (1), and Mark Claussen ${ }^{6}$ \\ ${ }^{1}$ Jet Propulsion Laboratory, MS 183-900, Caltech, Pasadena, CA 91109, USA; raghvendra.sahai@jpl.nasa.gov \\ ${ }^{2}$ Academia Sinica Institute of Astronomy and Astrophysics, P.O. Box 23-141, Taipei 106, Taiwan \\ ${ }^{3}$ Dpto. de Astrofísica Molecular e Infraroja, Instituto de Estructura de la Materia-CSIC, Serrano 121, E-28006 Madrid, Spain \\ ${ }^{4}$ Harvard-Smithsonian Center for Astrophysics, Cambridge, USA \\ ${ }^{5}$ Division of Astronomy \& Astrophysics, UCLA, Los Angeles, CA 90095-1547, USA \\ ${ }^{6}$ National Radio Astronomy Observatory, 1003 Lopezville Road, Socorro, NM 87801, USA \\ Received 2017 August 11; accepted 2017 October 5; published 2017 November 28
}

\begin{abstract}
We present interferometric and single-dish molecular line observations of the interstellar bullet-outflow source IRAS 05506+2414, whose wide-angle bullet spray is similar to the Orion BN/KL explosive outflow and likely arises from an entirely different mechanism than the classical accretion-disk-driven bipolar flows in young stellar objects. The bullet-outflow source is associated with a large pseudo-disk and three molecular outflows-a highvelocity outflow (HVO), a medium-velocity outflow (MVO), and a slow, extended outflow (SEO). The size (mass) of the pseudo-disk is $10,350 \mathrm{au} \times 6400 \mathrm{au}\left(0.64-0.17 M_{\odot}\right)$; from a model-fit assuming infall and rotation, we derive a central stellar mass of 8-19 $M_{\odot}$. The HVO $(\mathrm{MVO})$ has an angular size $\sim 5180(\sim 3330)$ au and a projected outflow velocity of $\sim 140 \mathrm{~km} \mathrm{~s}^{-1}\left(\sim 30 \mathrm{~km} \mathrm{~s}^{-1}\right)$. The SEO size (outflow speed) is $\left.\sim 0.9 \mathrm{pc}(\sim 6 \mathrm{~km} \mathrm{~s})^{-1}\right)$. The HVO's axis is aligned with (orthogonal to) that of the SEO (pseudo-disk). The velocity structure of the MVO is unresolved. The scalar momenta in the HVO and SEO are very similar, suggesting that the SEO has resulted from the HVO interacting with ambient-cloud material. The bullet spray shares a common axis with the pseudo-disk and has an age comparable to that of MVO (few hundred years), suggesting that these three structures are intimately linked. We discuss several models for the outflows in IRAS $05506+2414$ (including dynamical decay of a stellar cluster, chance encounter of a runaway star with a dense cloud, and close passage of two protostars), and conclude that second-epoch imaging to derive proper motions of the bullets and nearby stars can help to discriminate between them.
\end{abstract}

Key words: ISM: clouds - ISM: individual objects (IRAS05506+2414) - radio lines: ISM - stars: formation stars: mass-loss - stars: pre-main sequence

\section{Introduction}

High-mass $\left(M>8 M_{\odot}\right)$ stars play a decisive role in the evolution of galaxies; throughout their life-cycles, they inject large amounts of energy and momentum into their environments through massive stellar winds, UV radiation, and finally in their deaths as supernovae (Zinnecker \& Yorke 2007). In the earliest, very poorly understood, stages of their lives, massive stars and their immediate environments are characterized by high luminosities $\left(\gtrsim 10^{4} L_{\odot}\right)$, dense and warm molecular gas, strong far-infrared dust emission (e.g., Sridharan et al. 2002), and very weak or no free-free continuum emission at $\mathrm{cm}$ wavelengths (as they have not yet developed an ultracompact $\mathrm{H}$ II region).

Like their low-mass counterparts, high-mass protostars also exhibit bipolar outflows having varying degrees of collimation and outflow luminosities (Qiu et al. 2008). An exciting recent result in the understanding of this early evolutionary stage, based on a $\mathrm{CO} J=2-1$ interferometric mapping study of the Orion BN/KL region (Zapata et al. 2009), is that its enigmatic wide-angle outflow was produced by a violent explosion during the dynamical decay of a non-hierarchical massive young stellar system $\sim 500$ year ago (e.g., Bally et al. 2011: BCM11, Bally et al. 2017). A competing hypothesis is the chance encounter of a runaway star (BN) with the KL star-forming core (Tan 2004; Chatterjee \& Tan 2012).

These are entirely different mechanisms from the classical accretion-disk-driven bipolar flows in YSOs, and raise an important question: are the Orion $\mathrm{BN} / \mathrm{KL}$ explosive outflow and its engine unique, or simply the most prominent example of a relatively commonplace phenomenon? The latter scenario appears more likely, given that most massive stars form in dense clusters where dynamic interactions between the most massive members may be common, and 30\% of O-type stars are high-velocity runaways.

Our discovery, using $H S T$, of the second clear-cut example of this phenomenon in our Galaxy, IRAS 05506+2414 (hereafter IRAS 05506) (Sahai et al. 2008, Setal08), now provides a new opportunity to test these models. A few additional examples of the explosive outflow phenomenon are listed in BCM11 (e.g., DR21, Zapata et al. 2013), but they are somewhat less compelling and/or lack the detailed similarity with Orion $\mathrm{BN} / \mathrm{KL}$.

The HST optical and near-infrared images of IRAS 05506 show a bright, compact central source (Sa: Setal08) with a jet-like extension (Sa-jet: Setal08) and a fan-like spray of elongated knots that appear to emanate from it. Optical spectra show that these knots are moving at high velocities (radial velocity $350 \mathrm{~km} \mathrm{~s}^{-1}$ ) (Setal08). These structures are analogous to the near-IR "bullets" seen toward the BN/KL source in the Orion nebula. Interferometric observations of IRAS 05506 at $2.6 \mathrm{~mm}$ showed the presence of a highvelocity $\mathrm{CO}$ outflow (hereafter $\mathrm{HVO}$ ) aligned with the optical jet structure and roughly orthogonal to the bullet-spray. Setal08 also found extended $\mathrm{NH}_{3}(1,1)$ emission toward IRAS 05506 that, together with the combined presence of farIR, $\mathrm{H}_{2} \mathrm{O}$ and $\mathrm{OH}$ maser, $\mathrm{CO}$ and $\mathrm{CS} J=2-1$, emission, strongly suggested a dense, dusty star-forming core associated 
Table 1

Interferometric Observing Log and Main Observational Parameters

\begin{tabular}{|c|c|c|c|c|c|}
\hline Lines, Cont & $\begin{array}{l}\text { Freq } \\
(\mathrm{GHz})\end{array}$ & Array & $\begin{array}{c}\text { Beam (Maj } \times \text { Min }) \\
\left({ }^{\prime \prime}\right) \times\left({ }^{\prime \prime}\right)\end{array}$ & $\begin{array}{c}\text { PA } \\
\text { (degree) }\end{array}$ & Date \\
\hline \multicolumn{6}{|c|}{ SMA Observations } \\
\hline $\mathrm{CO} 2-1^{\mathrm{a}}$ & 230.538 & Subcompact & $7 ! .05 \times 4$ !. 11 & 71.49 & 2010 Mar 20 \\
\hline $\operatorname{CO} 2-1^{\mathrm{a}}$ & 230.538 & $\mathrm{Ext}+\mathrm{Sub}$ & 1 ". $62 \times 1$ "! 01 & $72: 97$ & $\ldots$ \\
\hline $\mathrm{SiO} 5-4(v=0)$ & 217.105 & Ext + Sub & 1 !. $74 \times 1$ × & $720^{\circ} 72$ & $\cdots$ \\
\hline SO $5,6-4,5$ & 219.949 & $\mathrm{Ext}+\mathrm{Sub}$ & $1 "$ "71 × 1 !"05 & 78.11 & $\ldots$ \\
\hline $\mathrm{CO} 1-0$ & 115.271 & $\mathrm{H}+\mathrm{E}+\mathrm{L}$ & $3 ! \prime 12 \times 2$ !" 68 & $-20^{\circ} 61$ & 2003 Oct 31 \\
\hline Continuum & 112 & $\mathrm{H}+\mathrm{E}+\mathrm{L}$ & $2 ! .96 \times 2 ! .78$ & -33.37 & 2003 Oct 31 \\
\hline
\end{tabular}

Note.

${ }^{\mathrm{a}}$ The ${ }^{13} \mathrm{CO}(220.398 \mathrm{GHz})$ and $\mathrm{C}^{18} \mathrm{O}(219.560 \mathrm{GHz})$ lines were also observed simultaneously; the beam sizes scale inversely with their frequencies.

with IRAS 05506. Setal08 concluded that IRAS 05506 is probably an intermediate-mass or massive protostar. The lack of radio continuum and the late $\mathrm{G}$-early $\mathrm{K}$ spectral type derived by Setal08 from the optical spectrum of this object (Sánchez Contreras et al. 2008) implied that this star is very young, because its temperature is still too low to provide sufficient UV flux for ionisation. The timescale of its bullet-outflow is very short (a few hundred years).

In this paper, we present a new study of IRAS 05506, based on extensive millimeter-wave observations of molecular lines, using both single-dish and high-angular resolution interferometric data. The plan of the paper is as follows. We first describe our observational results for the large-scale (arcminute) cloud structure in which IRAS 05506 is embedded, as seen in molecular-line and thermal dust emission observed with single apertures (Section 3.1). We then describe observational results, using interferometric facilities to probe the small-scale (arcsecond) structure around the central source in IRAS 05506 (Section 3.2). Next, in Section 4, we discuss the properties of the main physical components associated with IRAS 05506, as derived from the above data sets-these include a pseudo-disk and several outflows. In Section 5, we compare IRAS 05506 with the well-studied Orion BN/KL bullet-spray source, in the context of the published theoretical models that have been used to explain such sources. We present our conclusions in Section 6.

\section{Observations}

\section{1. $S M A$}

We obtained observations at $\lambda \sim 1.3 \mathrm{~mm}$ with the SMA, ${ }^{7}$ using the subcompact and extended array configurations. The subcompact track was obtained as "filler" (i.e., the observations were carried out sharing the track with another project), with phase center coordinates (J2000 R.A., decl.): 05:53:43.539, $+24: 14: 45.2$, using seven antennas, with system temperatures $T_{\text {sys }} \sim 150-200 \mathrm{~K}$. We shifted the phase center to 05:53:43.549, $+24: 14: 43.999$ in order to be consistent with the later extended tracks. The frequency coverage was $217-221 \mathrm{GHz}$ in the LSB, and $229-233 \mathrm{GHz}$ in the USB.

\footnotetext{
7 The Submillimeter Array is a joint project between the Smithsonian Astrophysical Observatory and the Academia Sinica Institute of Astronomy and Astrophysics, and is funded by the Smithsonian Institution and the Academia Sinica.
}

Additional observational details (beam size, orientation) are provided in Table 1.

The Doppler correction for LO frequency was based on an extragalactic source that was being observed for the main track, with which we shared our observations. This led to a shift of $+55 \mathrm{~km} \mathrm{~s}^{-1}$ in the central velocity for our source (IRAS 05506). Our visibility data were post-corrected using the task uti_doppler_fix in MIR-IDL ${ }^{8}$ for this velocity shift.

With the extended array, data were taken over two tracks, one on 2012 January 20 (with six antennas, on-source time $\sim 6.5 \mathrm{hr}$ ) and another on 2012 February 9 (with seven antennas, on-source time $\sim 8 \mathrm{hr}$ ). During the extended array observations in 2012 February, the atmospheric conditions were very good (atmospheric optical depth at $225 \mathrm{GHz}$ was around 0.03), and phase stability was also excellent. Because the system temperatures during January $20\left(T_{\text {sys }} \sim 200-350 \mathrm{~K}\right)$ were much higher than for February $9\left(T_{\text {sys }} \sim 130-240 \mathrm{~K}\right)$, we have not used the former.

Comparing the visibility amplitudes in the subcompact-array (hereafter "subcom") data with the extended-array (hereafter "ext") data, over similar $u-v$ spacings, we find the extended amplitudes to be weaker by a factor of 0.85 . Hence, the visibilities in the ext data were scaled upwards by a factor $1 / 0.85$ in order to make these data sets consistent, before combining them into a single data set (hereafter "extsub"), with high angular resolution $(\sim 1$." 4$)$ as well as sensitivity to extended structures on a scale of $\sim 10^{\prime \prime}$. In the rest of the paper, the default data cube used for presenting SMA observational results is the extsub one.

\subsection{OVRO}

We obtained observations at $\lambda \sim 2.6 \mathrm{~mm}$ with OVRO using the H, E, and L array configurations; data were obtained with two tracks for each of the arrays. The units of the digital spectral-line correlator were arranged to provide a total bandwidth of $90 \mathrm{MHz}\left(\sim 234 \mathrm{~km} \mathrm{~s}^{-1}\right)$, with a channel spacing of $1 \mathrm{MHz}$ (corresponding to $\sim 2.6 \mathrm{~km} \mathrm{~s}^{-1}$ ). The $3 \mathrm{~mm}$ continuum emission was observed simultaneously using the dualchannel analog continuum correlator, which provided a total bandwidth of $4 \mathrm{GHz}$ (after combining both IF bands). Additional observational details (beam size, orientation) are provided in Table 1.

\footnotetext{
8 https://www.cfa.harvard.edu/ cqi/mircook.html
} 
Table 2

Single-dish Observing Log and Main Observational Parameters

\begin{tabular}{|c|c|c|c|c|c|c|}
\hline Lines & $\begin{array}{c}\text { Freq } \\
(\mathrm{GHz})\end{array}$ & Tel. & $\begin{array}{l}T_{\text {sys }} \\
(\mathrm{K})\end{array}$ & $\eta_{b}{ }^{\mathrm{a}}$ & Backend & Date \\
\hline $\mathrm{CO} 1-0$ & 115.271 & ARO KP12m & 557 & 0.83 & $12 \mathrm{M}-\mathrm{MAC}^{\mathrm{b}}$ & 2007 Nov 18 \\
\hline $\mathrm{CO} 3-2$ & 345.796 & ARO SMT & 1716 & 0.70 & CTS & 2008 Feb 20 \\
\hline $\mathrm{CO} 4-3$ & 461.041 & $\mathrm{CSO}$ & 2213 & 0.53 & FFTS $^{\mathrm{d}}$ & 2013 Mar 06 \\
\hline${ }^{13} \mathrm{CO} 1-0$ & 110.201 & ARO KP12m & 207 & 0.83 & 12M-MAC & 2007 Nov 20 \\
\hline${ }^{13} \mathrm{CO} 2-1$ & 220.399 & ARO SMT & 163 & 0.74 & CTS & 2008 Feb 19 \\
\hline SO $4,3-3,2$ & 158.972 & ARO KP12m & 205 & 0.76 & 12M-MAC & 2008 Feb 28 \\
\hline SO $5,6-4,5$ & 219.949 & ARO SMT & 150 & 0.74 & CTS & 2008 Jan 26 \\
\hline SO $6,7-5,6$ & 261.844 & ARO SMT & 217 & 0.74 & CTS & 2008 Feb 19 \\
\hline $\mathrm{SO}_{2} 4(3,1)-4(2,2)$ & 221.965 & ARO SMT & 149 & 0.74 & CTS & 2008 Feb 18 \\
\hline $\mathrm{SO}_{2} 12(1,12)-11(1,11)$ & 255.553 & ARO SMT & 181 & 0.74 & CTS & 2008 Feb 19 \\
\hline $\mathrm{HCO}^{+} 1-0$ & 89.1895 & ARO KP12m & 322 & 0.89 & 12M-MAC & 2007 Nov 29 \\
\hline
\end{tabular}

Notes.

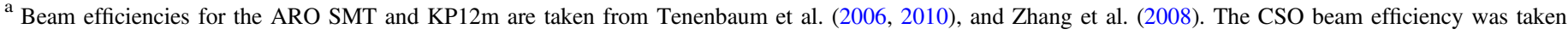
from http://www.submm.caltech.edu/cso/receivers/beams.html.

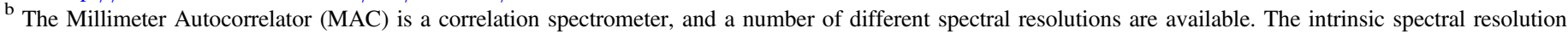
(bandwidth) was typically $24.4 \mathrm{kHz}(150 \mathrm{MHz})$. Spectra were post-processed to a lower spectral resolution to increase the $\mathrm{S} / \mathrm{N}$ as appropriate.

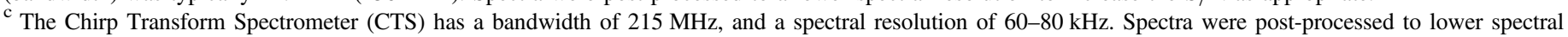
resolutions in order increase the $\mathrm{S} / \mathrm{N}$ as appropriate.

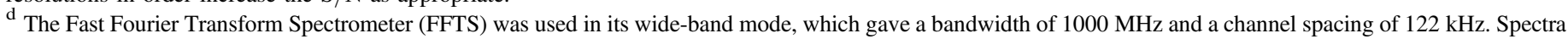
were post-processed to lower spectral spectral resolutions in order to increase the $\mathrm{S} / \mathrm{N}$ as appropriate.

The calibration of the data was performed using the MMA software package. ${ }^{9}$ Data were gain-calibrated in baseline-based mode using the quasar $\mathrm{J} 0530+135$, which was observed at regular time intervals of $\sim 20$ minutes before and after our target. The quasars 3C 273 and 3 C 84 were used as passband and flux calibrators.

Reconstruction of the maps from the visibilities was done using the Multichannel Image Reconstruction, Image Analysis and Display (MIRIAD) software. We Fourier transformed the measured visibilities with robust weighting (which is an optimized compromise between natural and uniform weighting) for ${ }^{12} \mathrm{CO}$ and natural weighting for the continuum map for $\mathrm{S} / \mathrm{N}$ optimization.

\subsection{Single-dish Observations}

We obtained single-dish observations of a variety of molecular lines (listed in Table 2) toward IRAS 05506 using the Arizona Radio Observatory's (ARO) SMT-10 m and Kitt Peak $12 \mathrm{~m}$ (KP12m) telescopes, and the Caltech Submillimeter Observatory's (CSO) $10 \mathrm{~m}$ telescope. These "on-source" spectra were obtained toward the coordinates of the optical/ infrared source in IRAS 05506 (i.e., Sa) using coordinates (J2000 R.A., decl.): 5:53:43.6, 24:14:45, by position-switching the telescopes using an emission-free reference position. The beam sizes (FWHM) for the SMT-10 $\mathrm{m}$ at nominal frequencies of 345 and $230 \mathrm{GHz}$ are $32^{\prime \prime}$ and $22^{\prime \prime}$, respectively. For the $\mathrm{KP} 12 \mathrm{~m}(\mathrm{CSO})$, at $115(461) \mathrm{GHz}$, the beam size is 55" (16". 5). The beam sizes scale with the wavelength over broad

\footnotetext{
9 MMA is written and maintained by Caltech's Millimeter Interferometry Group.
}

wavelength ranges within the $0.65,0.8,2.6$ and $1.3 \mathrm{~mm}$ windows.

We mapped the ${ }^{12} \mathrm{CO}$ and ${ }^{13} \mathrm{CO} \quad J=2-1$ emission simultaneously toward a $\sim 11 ! 2 \times 5.2$ area centered on IRAS 05506 using the SMT's On-The-Fly (OTF) mapping observational mode (see, e.g., Bieging \& Peters 2011 for details of the technique).

All data were calibrated using the standard chopper calibration method. Telescope pointing was checked frequently, i.e., every 45-60 minutes-at the ARO, we used Mars as a continuum pointing source, and at the CSO, we used the circumstellar shell of the evolved star GX Mon as a line (CO $J=2-1)$ pointing source. Additional relevant observing details (e.g., system temperatures, spectrometer backends, beam efficiencies) are given in Table 2.

\section{Results}

\subsection{Single-dish Data: The Large-scale Environment of IRAS 05506}

\subsubsection{CO Observations}

The on-source ${ }^{12} \mathrm{CO} J=2-1$ and $1-0$ lines show a doublepeaked core, with a central minimum at $V_{\mathrm{lsr}} \sim 6 \mathrm{~km} \mathrm{~s}^{-1}$, and prominent wings (Figure 1). The on-source ${ }^{12} \mathrm{CO} J=3-2$ and 4-3 lines show an emission peak near $V_{\mathrm{lsr}} \sim 7.4 \mathrm{~km} \mathrm{~s}^{-1}$, with a FWHM velocity width of $\sim 2 \mathrm{~km} \mathrm{~s}^{-1}$, as well as a weaker, significantly broader component, having a FWHM of roughly 10 (15) $\mathrm{km} \mathrm{s}^{-1}$. In contrast, the corresponding ${ }^{13} \mathrm{CO} 2-1$ and $1-0$ line profiles are centrally peaked at $V_{\mathrm{lsr}} \sim 6 \mathrm{~km} \mathrm{~s}^{-1}$, with a $\mathrm{FWHM}=1.5 \mathrm{~km} \mathrm{~s}^{-1}$. 


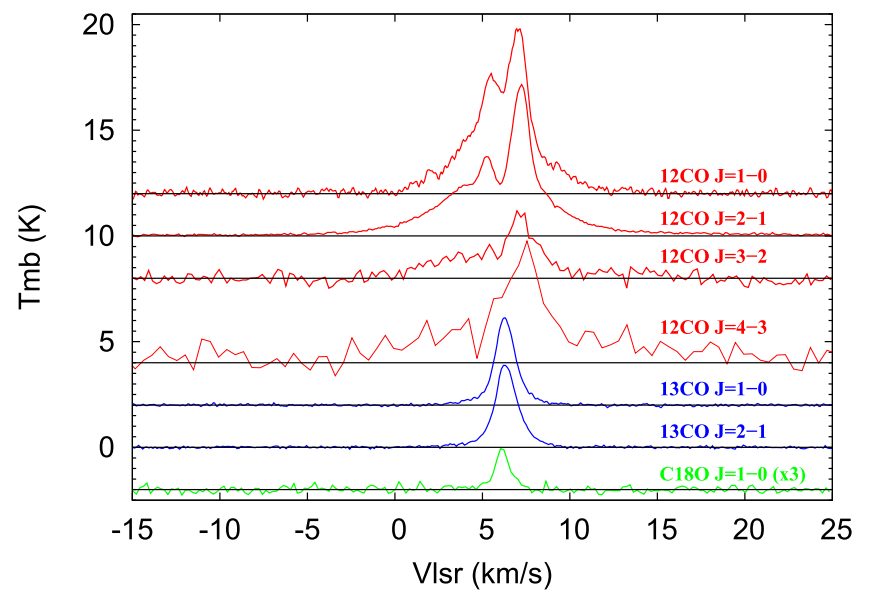

Figure 1. Single-dish observations of IRAS 05506 in the millimeter-wave lines from CO and its isotopologues, using the ARO's Kitt Peak 12 m, SMT, and the CSO. Different transitions are shown in different colors and shifted vertically for clarity. The beam sizes are different for different lines (Section 2.3).

Our OTF mapping shows that ${ }^{12} \mathrm{CO} J=2-1$ emission is very extended, and at around $V_{\mathrm{lsr}} \sim 6 \mathrm{~km} \mathrm{~s}^{-1}$, it covers the full extent of the mapped area (i.e., $\sim 11 ! 2 \times 5$. 2 ) at the $7.5 \sigma$ noise level (1 K intensity) (Figure 2$)$. We will refer to this cloud as "cloud A"; this cloud is likely a substructure of an even larger cloud, extending $30^{\prime} \mathrm{EW}$ and $10^{\prime} \mathrm{NS}$, that was mapped by Setal08 in ${ }^{12} \mathrm{CO} J=1-0$ around IRAS 05506 with ARO's $\mathrm{KP} 12 \mathrm{~m}$ dish. The ${ }^{13} \mathrm{CO} J=2-1$ emission at $V_{\mathrm{lsr}} \sim 6 \mathrm{~km} \mathrm{~s}^{-1}$ is much less extended (Figure 3 ) and is strongly peaked toward IRAS 05506 - the contour at $30 \%$ of the peak ${ }^{13} \mathrm{CO}$ intensity defines a roughly triangular-shaped region (with east-west and north-south aligned sides of length $\sim 125^{\prime \prime}$ ). We will refer to this structure as "cloud A core".

The on-source $\mathrm{C}^{18} \mathrm{O} J=1-0$ line profile has the narrowest width $\left(\mathrm{FWHM} \sim 0.4 \mathrm{~km} \mathrm{~s}^{-1}\right.$ ) among all the single-dish $\mathrm{CO}$ line profiles, and is likely dominated by dense, cold gas in the cloud A core, whereas the other ${ }^{12} \mathrm{CO}$ and ${ }^{13} \mathrm{CO} J=1-0$ line profiles likely include contributions from an outflow (see below).

Using the OTF, we find that the emissions in the blue $\left(V_{\mathrm{lsr}}=0-5 \mathrm{~km} \mathrm{~s}^{-1}\right)$ and red wings $\left(7-12 \mathrm{~km} \mathrm{~s}^{-1}\right)$, as seen in the on-source ${ }^{12} \mathrm{CO}(2-1)$ profile, delineate two large extended bipolar outflow lobes around the location of IRAS 05506, with the blue (red)-shifted lobe located SW (NE) of center (Figure 4). Hereafter, we refer to this outflow as the Slow Extended Outflow, or SEO. We do not find emission in our OTF map at velocities shortward (longward) of $V_{\mathrm{lsr}} \sim 0 \mathrm{~km} \mathrm{~s}^{-1}$ $\left(V_{\mathrm{lsr}} \sim 12 \mathrm{~km} \mathrm{~s}^{-1}\right)$.

\subsubsection{Shock and High-density Tracers}

Out of the four SO lines observed $(\mathrm{N}, J=6,7-5,6, N$, $J=5,6-4,5, N, J=4,3-3,2$ and $N, J=3,4-2,3$ : hereafter SO lines 1, 2, 3, and 4, respectively), two (SO lines 2 and 3) show a narrow central component, together with weak wings. The highest-excitation line, SO line 4, shows only the broad component, and the lowest-excitation line, SO line $1^{10}$, shows only the narrow component (Figure 5). We also detected weak, broad emission from the $\mathrm{SO}_{2} J\left(K_{a}, K_{c}\right)=4(3,1)-4(2,2)$ and $12(1,12)-11(1,11)$ lines. The $\mathrm{SO}$ and $\mathrm{SO}_{2}$ emissions likely

\footnotetext{
10 The energies of the upper levels for SO lines $1-4$ are, respectively, 11.02 , $19.93,24.30$, and $33.04 \mathrm{~cm}^{-1}$.
}

arise from shocked gas, as has been proposed in the case of the HH 212 jet (Podio et al. 2015).

The $\mathrm{HCO}^{+} J=3-2$ line profile has a central narrow component with FWHM $1.3 \mathrm{~km} \mathrm{~s}^{-1}$, and a broad component with wings extending to $\sim-15 \mathrm{~km} \mathrm{~s}^{-1}$ and $\sim 30 \mathrm{~km} \mathrm{~s}^{-1}$; the $\mathrm{HCO}^{+} J=1-0$ line shows only the narrow component. We show later that the broad velocity wings of the SO lines likely arise in a very compact outflow in IRAS 05506 (Section 3.2.2). The $\mathrm{SO}_{2}$ and $\mathrm{HCO}^{+}$broad-wing emissions, which cover a similar velocity range as $\mathrm{SO}$, most likely also come from the same outflow.

The CS $J=3-2$ and $\mathrm{H}_{2} \mathrm{CO} 2(1,1)-1(1,0)$ lines show only a narrow component centered at $V_{\mathrm{lsr}}=6.3 \mathrm{~km} \mathrm{~s}^{-1}$, which most likely comes from dense gas in the cloud A core.

\subsubsection{Continuum}

IRAS 05506 was observed with the SPIRE and PACS instruments onboard the Herschel Space Observatory (HSO), as part of the Hi-Gal survey (Molinari et al. 2016). We have downloaded the Level 2.5 images from the Herschel Science Archive. The SPIRE images (at 500, 350, and $250 \mu \mathrm{m}$ ) show a bright emission source roughly centered at the location of the 2MASS optical/infrared source in IRAS 05506 (Sa) found by Setal08 $^{11}$ (Figure 6). The far-IR source has a morphology that is similar to that of the ${ }^{13} \mathrm{CO} J=2-1$ emission from the cloud A core. Much fainter dust nebulosity can be seen extending roughly toward the ENE and W, with the same extent and morphology as the faint emission seen in the ${ }^{12} \mathrm{CO} J=2-1$ emission at the radial velocity of cloud A. The central peak appears to be elongated SE-NW (Figure 6(d), inset). The halfpower size of this peak is $31^{\prime \prime} \times 21^{\prime \prime}$ (deconvolved size is $25^{\prime \prime} \times 11$." 5 , given the 17!" 6 SPIRE beam at $\left.250 \mu \mathrm{m}\right)$.

The PACS images (70 and $160 \mu \mathrm{m})$ show emission predominantly from a bright, round source with a half-power diameter of $10 . \prime 8$ at $70 \mu \mathrm{m}$, centered at the location of $H S T$ source Sa (deconvolved size is 9!"3, given the $\sim 5$ ! 4 PACS beam at $70 \mu \mathrm{m})$.

\subsection{Interferometric Data: the Central Source of IRAS 05506}

We detected emission in the $J=2-1$ lines of ${ }^{12} \mathrm{CO},{ }^{13} \mathrm{CO}$, and $\mathrm{C}^{18} \mathrm{O}$, and the SO $N, J=5,6-4,5$, and $\mathrm{SiO} J=5-4$ $(v=0)$ lines from IRAS 05506 with the SMA. Additional tracks were observed toward IRAS 05506 with the OVRO array ${ }^{12}$, more extended than those presented in Setal08 for the ${ }^{12} \mathrm{CO}$ and ${ }^{13} \mathrm{CO} J=1-0$ lines.

\subsubsection{CO Observations}

IRAS $05506(\mathrm{Sa})$ is located near the NE periphery of an extended, structured cloud of size $\sim 15^{\prime \prime} \times 7^{\prime \prime}$ - the latter is best seen in the ${ }^{13} \mathrm{CO}$ and $\mathrm{C}^{18} \mathrm{O} J=2-1$ maps, because these lines are more optically thin than ${ }^{12} \mathrm{CO} J=2-1$ (Figure 7(a), (b)). This cloud (hereafter "cloud B") has its long axis oriented roughly along the SE-NW direction, as seen in the ${ }^{13} \mathrm{CO}$ $J=2-1$ map at $V_{\mathrm{lsr}}=6 \mathrm{~km} \mathrm{~s}^{-1}$.

We note that cloud $\mathrm{B}$ shows several distinct compact peaks in the ${ }^{13} \mathrm{CO}$ and $\mathrm{C}^{18} \mathrm{O} J=2-1$ maps at $V_{\mathrm{lsr}}=5-7 \mathrm{~km} \mathrm{~s}^{-1}$, none of which are associated with the IRAS $05506 \mathrm{Sa}$. These

\footnotetext{
11 J2000 R.A., decl.: 05:53:43.56, 24:14:44.7.

12 During the course of a survey of post-AGB objects by Sánchez Contreras \& Sahai (2012).
} 


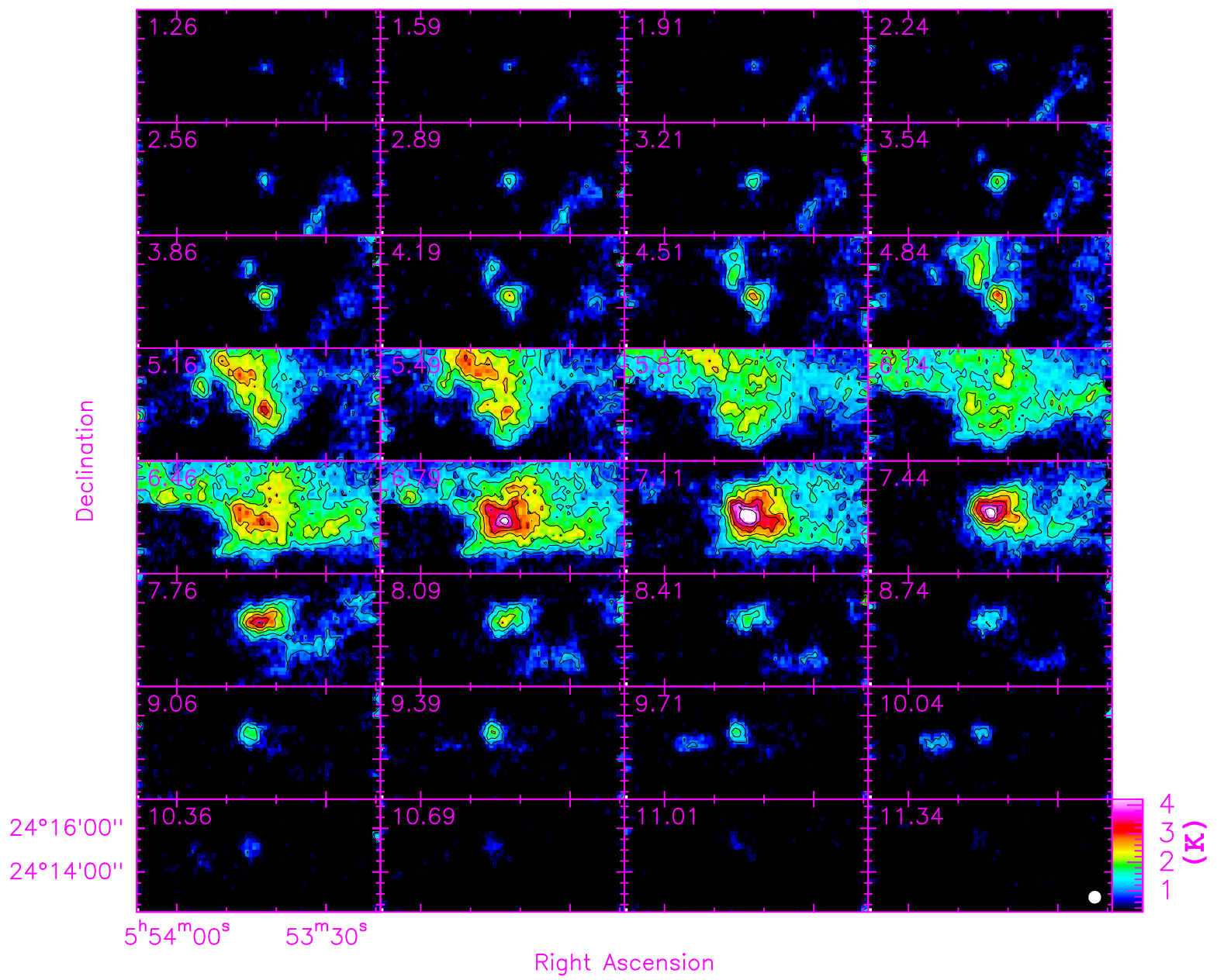

Figure 2. SMT OTF channel map of ${ }^{12} \mathrm{CO} J=2-1$ emission from IRAS 05506 and its large-scale environment, covering a $11 ! 2 \times 5 ! 2$ area. Each channel is $0.325 \mathrm{~km} \mathrm{~s}^{-1}$ wide, the beam (white circle in lower-right panel) is $32^{\prime \prime}$ (FWHM), and the contour levels are 0.5, 1.0, 1.5, 2.0, 2.5, 3.0, and 3.5 K.

compact cores (the brightest of these are labelled $P k_{a}$ and $P k_{b}$ ) show no evidence for stars, as no associated point-sources are found in the near-IR (HST $0.8 \mu$ mimage, Setal08) or IR (2MASS J,H, K-band images, Setal08) or an archival Spitzer/IRAC $4.5 \mu \mathrm{m}$ image. In the 2MASS and Spitzer images, the wings of the PSF of the very bright IRAS 05506 source at these wavelengths overlap the locations of these dense cores, making it difficult to discern faint counterparts. However, this is not an issue in the HST image, and although faint stars can be seen even closer to the bright IRAS 05506 source than $P k_{a}$ and $P k_{b}$ (Setal08), there are no optical counterparts for these peaks. There is also no evidence for broad-velocity emission signifying the presence of outflows associated with these peaks.

At the location of IRAS 05506, no emission peak is seen in the ${ }^{13} \mathrm{CO} J=2-1$ map at $V_{\mathrm{lsr}} \sim 6 \mathrm{~km} \mathrm{~s}^{-1}$, but when the emission is integrated over a broad velocity range, $V_{\mathrm{lsr}}=-4.5$ to $18.5 \mathrm{~km} \mathrm{~s}^{-1}$ (Figure 7(c)), a bright compact source becomes visible there. Channel maps of the ${ }^{12} \mathrm{CO} J=2-1$ emission, covering a central velocity range around IRAS 05506's systemic velocity, i.e., $-9 \leqslant V_{\mathrm{lsr}}\left(\mathrm{km} \mathrm{s}^{-1}\right) \leqslant 20$, show emission mostly confined to a rather compact structure, about 2 ". 5 in size, centered at coordinates (J2000 R.A., decl.): 05:53:43.55, 24:14:45.1 (Figure 8), except in the $V_{\mathrm{lsr}}=5$ and $6 \mathrm{~km} \mathrm{~s}^{-1}$ channels, where the emission is very weak or absent.
This absence of emission results from strong absorption by cold gas in cloud A (described above, Section 3.1.1).

A similar compact source is seen in the OVRO ${ }^{12} \mathrm{CO} J=1-0$ map at the same location (Figure 9), but appears slightly more extended because of the larger beam size in these data. However, unlike $J=2-1$, the $J=1-0$ data show the compact source also in the $V_{\mathrm{lsr}}=6.5 \mathrm{~km} \mathrm{~s}^{-1}\left(2.6 \mathrm{~km} \mathrm{~s}^{-1}\right.$ wide) channel, which overlaps the velocity range of the $V_{\mathrm{lsr}}=5$ and $6 \mathrm{~km} \mathrm{~s}^{-1}$ channels in the SMA map. We infer that the foreground cloud $A$ has a significantly lower optical depth in ${ }^{12} \mathrm{CO} J=1-0$, compared to $J=2-1$, along the line of sight toward the compact source. The compact source in IRAS 05506 is not seen in $\mathrm{C}^{18} \mathrm{O} J=2-1$ or ${ }^{13} \mathrm{CO} J=1-0$, either at the systemic velocity or when integrated over a broad-velocity range (as above, $V_{\mathrm{lsr}}=-4.5$ to $18.5 \mathrm{~km} \mathrm{~s}^{-1}$ ), and we conclude that the emission from the source in the lines of $\mathrm{CO}$ and its isotopologues is dominated by relatively optically thin emission extending over a broad velocity range.

In order to understand the kinematic structure of this compact $\mathrm{CO}$ source, we have extracted a ${ }^{12} \mathrm{CO} J=2-1$ spectrum from the extsub data cube using a $10^{\prime \prime} \times 10^{\prime \prime}$ boxaperture centered on IRAS 05506. The spectrum shows a triplepeaked structure in its core (Figure 10(a)), which is a combination of two features: the first is a broad absorption feature extending over $V_{\mathrm{lsr}} \sim 3-10 \mathrm{~km} \mathrm{~s}^{-1}$ due to foreground absorption from cloud A (and/or cloud A core), and the second is emission in the $V_{\mathrm{lsr}} \sim 7-8 \mathrm{~km} \mathrm{~s}^{-1}$ range from an extended 


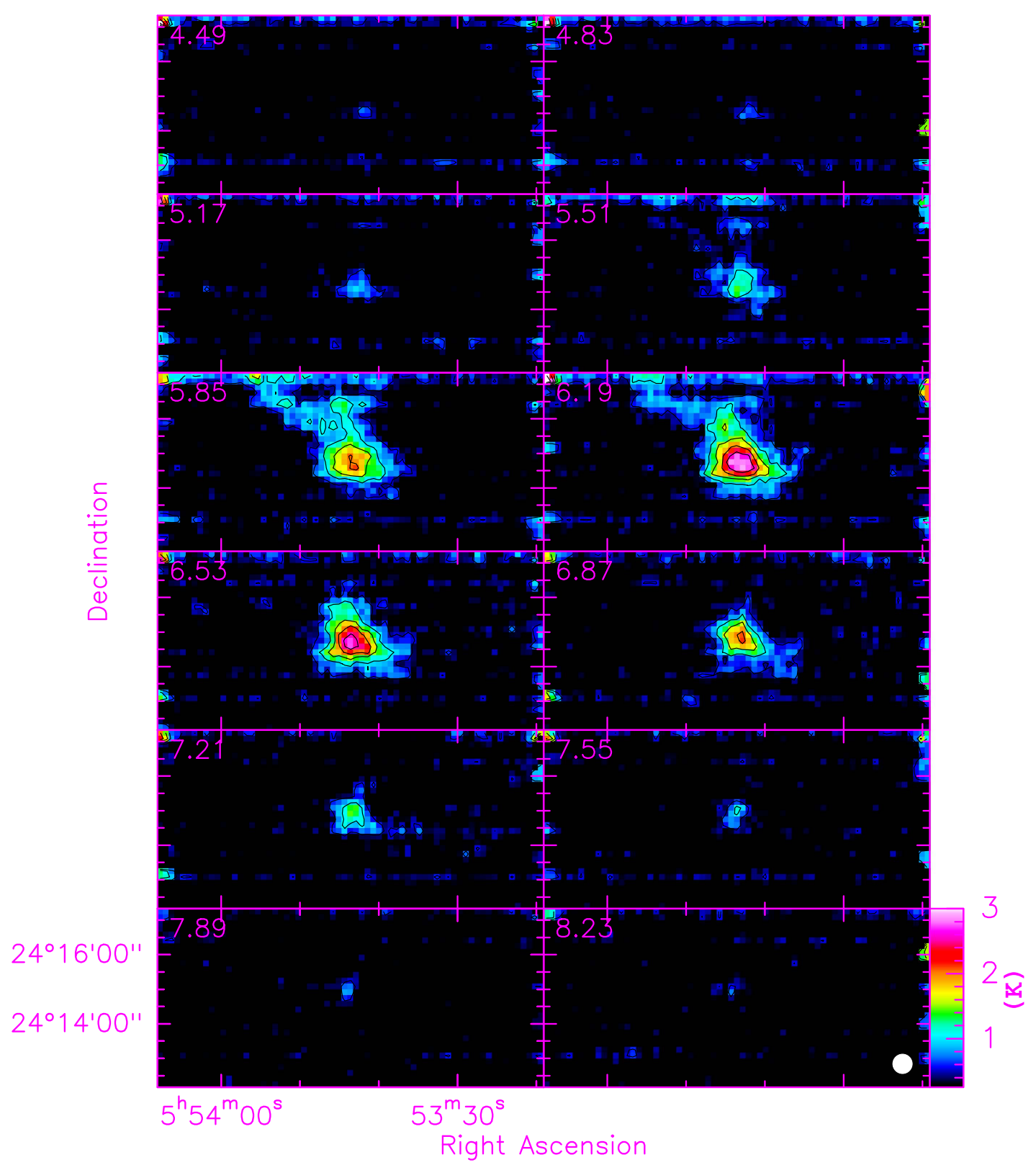

Figure 3. SMT OTF channel map of ${ }^{13} \mathrm{CO} J=2-1$ emission from IRAS 05506 and its large-scale environment, covering a 11!2 $\times 5$ ! 2 area (as in Figure 2). Each channel is $0.34 \mathrm{~km} \mathrm{~s}^{-1}$ wide, the beam (white circle in lower-right panel) is 33 !" 5 (FWHM), and contour levels are $0.5,1.0,1.5,2.0$, and $2.5 \mathrm{~K}$.

structure evident in the ${ }^{12} \mathrm{CO}$ channel map at these velocities (see Figure 8) (hereafter "cloudlet B1").

The ${ }^{13} \mathrm{CO} J=2-1$ spectrum does not show the triple-peaked structure seen in ${ }^{12} \mathrm{CO} J=2-1$. It is centrally peaked with a narrow core of FWHM $1.8 \mathrm{~km} \mathrm{~s}^{-1}\left(1.5 \mathrm{~km} \mathrm{~s}^{-1}\right.$ deconvolved), a broad component extending about $\pm 10 \mathrm{~km} \mathrm{~s}^{-1}$ from the peak at $V_{\mathrm{lsr}}=6-7 \mathrm{~km} \mathrm{~s}^{-1}$. The broad component comes from the compact source identified above in the ${ }^{13} \mathrm{CO} J=2-1$ moment 0 map (Figure 7(c)).

The ${ }^{12} \mathrm{CO}$ spectrum shows very extended wings seen out to $V_{\mathrm{lsr}}$ of $\sim-155 \mathrm{~km} \mathrm{~s}^{-1}$ in the blue wing and $\sim 120 \mathrm{~km} \mathrm{~s}^{-1}$ in the red wing (Figure 10(b)). In order to elucidate the structure of the faint emission in the line wings at $V_{\mathrm{lsr}}<-20 \mathrm{~km} \mathrm{~s}^{-1}$ and $V_{\mathrm{lsr}}>40 \mathrm{~km} \mathrm{~s}^{-1}$, we plot the integrated emission in selected velocity intervals covering the line wings (Figure 11). The strongest blueshifted and redshifted emission ${ }^{12} \mathrm{CO}$ $J=2-1$ emission at large velocity offsets from the systemic velocity (i.e., $V_{\mathrm{lsr}}=-52$ to $-20 \mathrm{~km} \mathrm{~s}^{-1}$ in the blue and $V_{\mathrm{lsr}}=40$ to $72 \mathrm{~km} \mathrm{~s}^{-1}$ in the red) is separated by $\sim 00^{\prime \prime} 9$ along an axis that is roughly oriented along PA $\sim 40^{\circ}$, with the blueshifted emission in the SW. At more extreme velocity offsets (i.e., $V_{\mathrm{lsr}}<-52 \mathrm{~km} \mathrm{~s}^{-1}$ and $V_{\mathrm{lsr}}>72 \mathrm{~km} \mathrm{~s}^{-1}$ ), the orientation of the vector joining the blue- and redshifted emission shifts to a larger PA, $\sim 55^{\circ}$, but the uncertainty in this value is large because the redshifted emission at these velocities is very weak, and because a weaker, secondary redshifted peak is found located to the SW of the blueshifted peak by $\sim 1$ "' 6 . We conclude that, although the HVO direction in the SMA ${ }^{12} \mathrm{CO} J=2-1$ data is roughly consistent with that inferred by Setal08 from their OVRO ${ }^{12} \mathrm{CO} J=1-0$ data, the $\mathrm{HVO}$ is likely more complex than a single bipolar outflow.

At small offset velocities $\left( \pm 10 \mathrm{~km} \mathrm{~s}^{-1}\right)$ relative to the systemic velocity, we find a structure that is kinematically different from the HVO. We find that the intensity peaks 


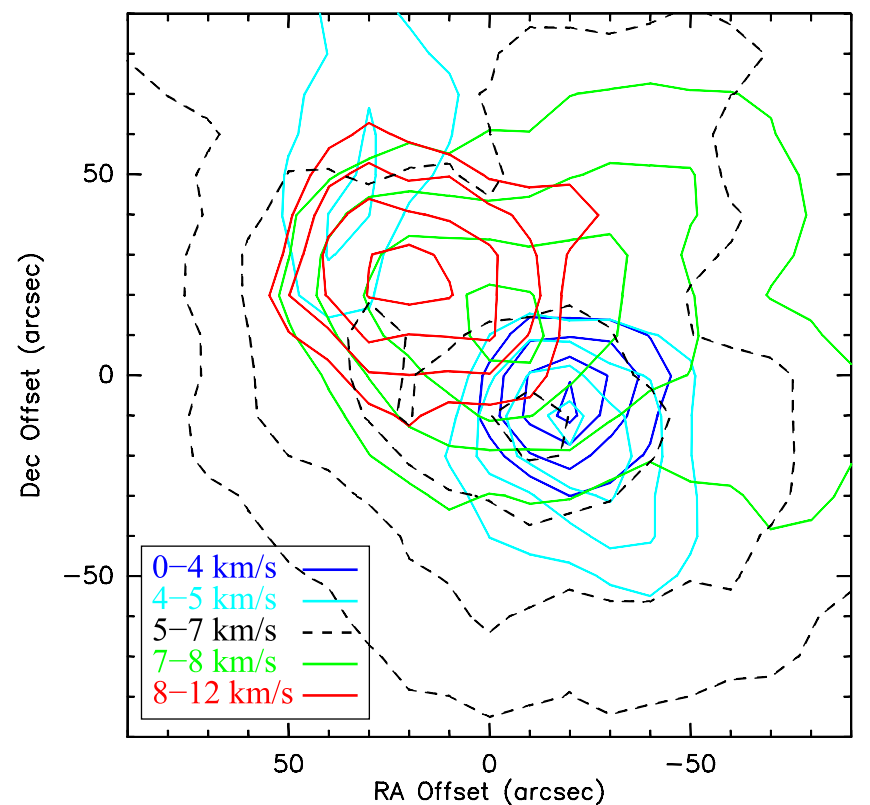

Figure 4. On-The-Fly (OTF) map of the ${ }^{12} \mathrm{CO} J=2-1$ emission toward IRAS 05506, using the SMT $10 \mathrm{~m}$, integrated over different velocity bins, showing the large-scale slow bipolar outflow (SEO). The beam size is $32^{\prime \prime}$. The map center is at (J2000 R.A., decl.): 5:53:43.6, 24:14:45. The bins are $V_{\mathrm{lsr}}=0-4 \quad \mathrm{~km} \mathrm{~s}^{-1}$ (blue), 4-5 $\mathrm{km} \mathrm{s}^{-1}$ (cyan), 5-7 $\mathrm{km} \mathrm{s}^{-1}$ (black), $7-8 \mathrm{~km} \mathrm{~s}^{-1}$ (green), and $8-12 \mathrm{~km} \mathrm{~s}^{-1}$ (red), and the contour levels are at $0.5,0.65,0.8$, and 0.95 of the peak integrated-intensity values for each velocity range $\left(5.1,2.7,7.2,4.0\right.$, and $4.5 \mathrm{~K}-\mathrm{km} \mathrm{s}^{-1}$, respectively).

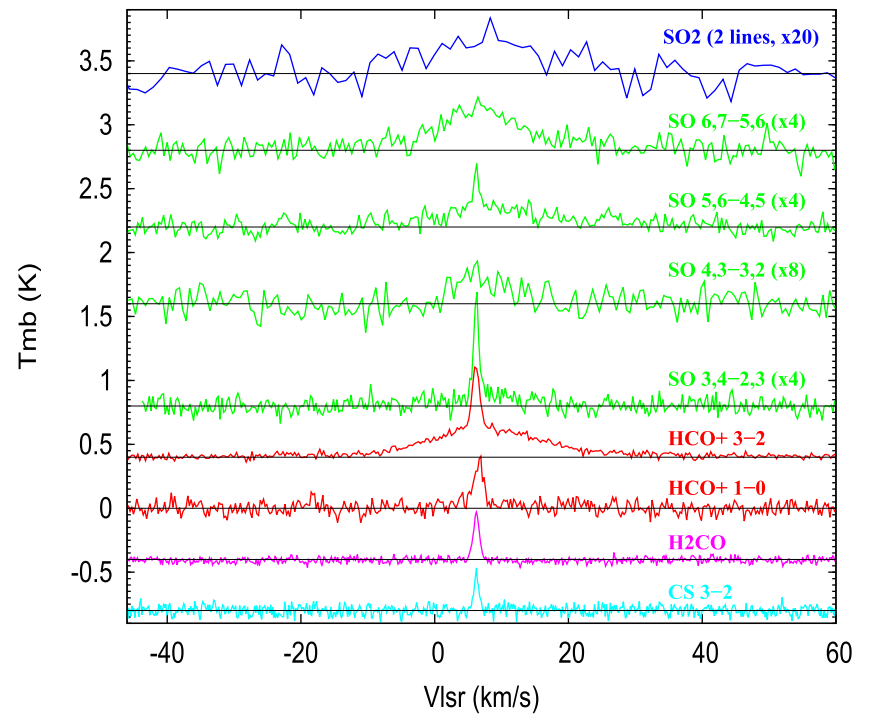

Figure 5. Single-dish observations of IRAS 05506 in the millimeter-wave lines that are probes of shocks and high-density gas, using the ARO's Kitt Peak $12 \mathrm{~m}$ and SMT $10 \mathrm{~m}$ telescopes. Different transitions are shown in different colors, scaled and shifted vertically for clarity. The $\mathrm{SO}_{2}$ spectrum shown is an average of the two weak $\mathrm{SO}_{2}$ lines (see Table 2) observed in IRAS 05506. The beam sizes are different for different lines (Section 2.3).

at 2.7 and $10.9 \mathrm{~km} \mathrm{~s}^{-1}$ in the ${ }^{12} \mathrm{CO} J=2-1$ line profile (Figure 10(a)), are separated spatially by 0.78 along an axis at $\mathrm{PA} \sim 40^{\circ}$ in our highest-resolution data (i.e., the ext data cube, with a beam of 1 "! $34 \times 0 . ! 81$ at $\mathrm{PA}=81.46$ ). A positionvelocity plot of a cut along this axis shows that the low-velocity emission is suggestive of differential rotation (Figure 12), such that the NE side is moving toward us, and the SW side is moving away from us. This gradient is opposite to that seen for the HVO emission. We label the structure producing this emission as the pseudo-disk (a model for the pseudo-disk in Section 4.2.1). We infer that the axis of this pseudo-disk, orthogonal to the direction of maximum velocity gradient above, is oriented at $\mathrm{PA} \sim 130^{\circ}$.

However, the kinematics of this structure is likely more complex than simply differential rotation, because in the latter case, the separation of the red- and blueshifted emission should become progressively smaller for increasingly larger offset velocities from the central velocity, but in the PV-plot shown in Figure 12, there appears to be a net separation even at the highest velocities.

A position-velocity plot of a cut along the pseudo-disk major axis extracted from the ${ }^{13} \mathrm{CO} J=2-1$ ext data cube (not shown) also shows a spatially compact emission structure extending over a velocity range $-4<V_{\mathrm{lsr}}<15 \mathrm{~km} \mathrm{~s}^{-1}$ that appears similar to the structure seen in the ${ }^{12} \mathrm{CO} J=2-1 \mathrm{PV}$ plot; we conclude that this emission also comes from the pseudo-disk.

\subsubsection{Shock and High-density Tracers}

The SO $N, J=5,6-4,5$ map at the peak emission velocity $\left(V_{\mathrm{lsr}}=6 \mathrm{~km} \mathrm{~s}^{-1}\right)^{13}$ shows that most of the emission arises from a compact source - the FWHM size of the core in the SO ext image (Figure 13(a)) is $\sim 1{ }^{\prime \prime} 8 \times 1 . \prime 5, \quad \mathrm{PA} \sim 100^{\circ}$ ). The emission peak is located at coordinates (J2000 R.A., decl.): 5:53:43.565, 24:14:44.68. Hence, given the FWHM beam size $\sim 1$." $46 \times 0$." 85 ( $\mathrm{PA}=82.5)$, the SO source is marginally resolved, with a deconvolved size of about $\sim 1$ !" $05 \times 1$ !' 2 . The $\mathrm{SiO} 5-4(v=0)$ emission map shows similar properties to $\mathrm{SO}$ $N, J=5,6-4,5$, but is weaker and therefore has lower signalto-noise. The $\mathrm{SO}$ and $\mathrm{SiO}$ line profiles extracted from apertures twice the FWHM source sizes in these lines (thus capturing almost all of the source flux), have roughly triangular shapes, with clearly visible wings covering about $60 \mathrm{~km} \mathrm{~s}^{-1}$ (from $V_{\text {lsr }} \sim-20$ to $40 \mathrm{~km} \mathrm{~s}^{-1}$ ) (Figure 13(b)).

Position-velocity plots of the intensity along a cut at $\mathrm{PA}=40^{\circ}$ in the $\mathrm{SO} N, J=5,6-4,5$ ext maps show weak evidence for a velocity gradient along $\mathrm{PA} \sim 40^{\circ}$ over the velocity range $-6<V_{\mathrm{lsr}}<20 \mathrm{~km} \mathrm{~s}^{-1}$ (Figure 14), which has the same sense as that seen in the pseudo-disk. We therefore conclude that some fraction of the emission seen in the SO line in the velocity range $-6 \gtrsim V_{\mathrm{lsr}} \gtrsim 20$ arises in the pseudo-disk.

The $\mathrm{SO}$ and $\mathrm{SiO}$ profiles are asymmetric about the central peak, with the redshifted side being brighter and broader than the blue one. Such a shape is a characteristic signature of selfabsorption in an optically thick outflow with a radially decreasing temperature distribution. We propose that some or all of the high-velocity wing emission, i.e., at velocities offset by $\gtrsim 12-15$ from the systemic velocity arises in an outflow (hereafter "medium-velocity outflow" or MVO). We do not find a convincing velocity gradient for this outflow because the SO emission is only marginally resolved in our maps. The asymmetry of the line profiles from the MVO is opposite to that seen for the emission from the $\mathrm{HVO}$ - for the latter, the ${ }^{12} \mathrm{CO}$ emission from the blueshifted component $\left(V_{\mathrm{lsr}}<\right.$ $\left.-20 \mathrm{~km} \mathrm{~s}^{-1}\right)$ is stronger than the redshifted one $\left(V_{\mathrm{lsr}}>40\right.$ $\mathrm{km} \mathrm{s}^{-1}$ ) (see Figure 10(b)). The ${ }^{12} \mathrm{CO}$ profile, in the velocity range of the MVO, has the same asymmetry as seen in the SO

\footnotetext{
${ }^{13}$ A map of the emission integrated over the line-width is similarly compact (not shown).
} 

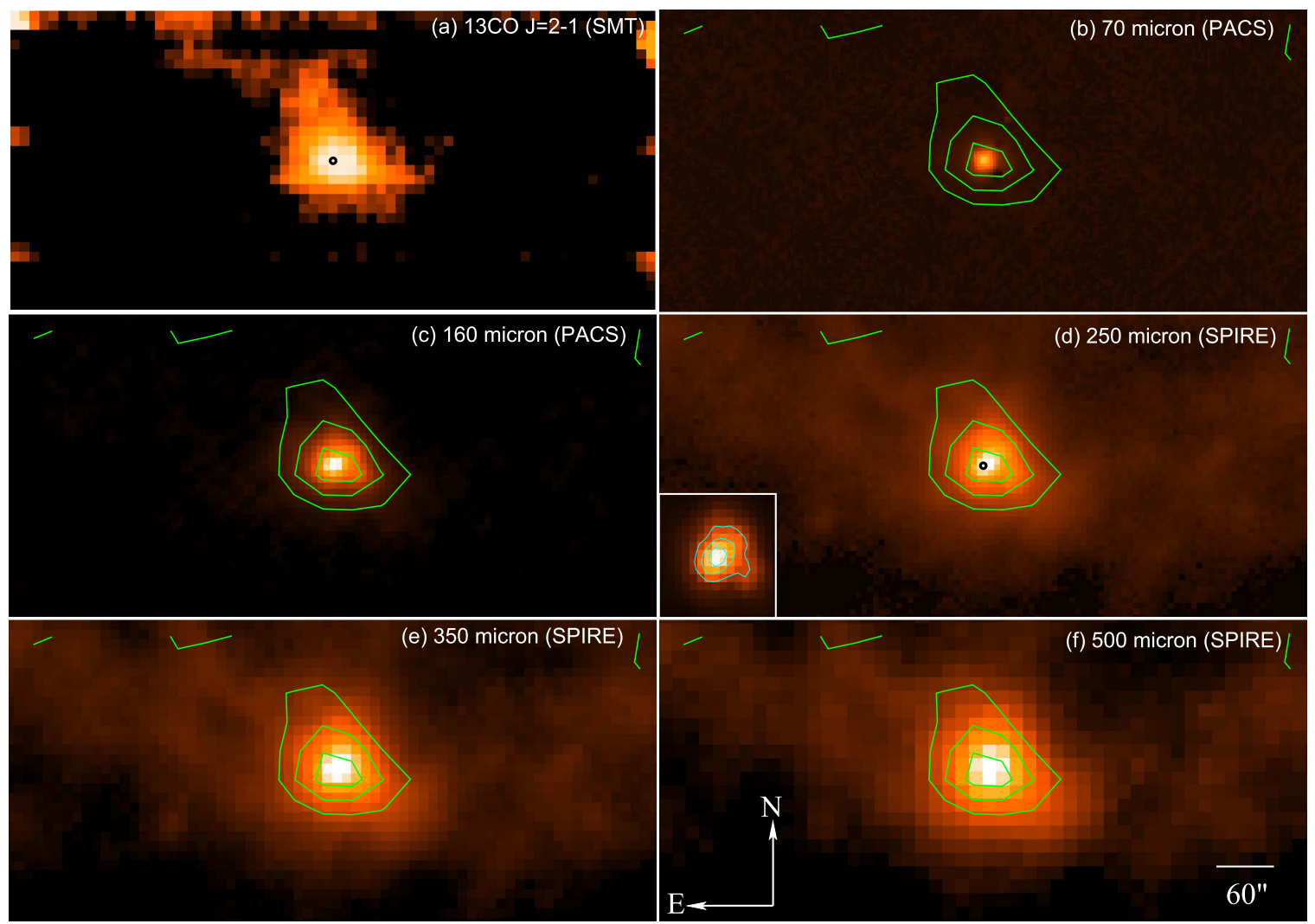

Figure 6. IRAS 05506 and its large-scale environment imaged with the SMT and Herschel, covering a 11!2 $\times 5$ '.2 area (as in Figure 2). (a) SMT ${ }^{13} \mathrm{CO} J=2-1$ map, (b, c) PACS 70 and $160 \mu \mathrm{m}$ image, (d, e, f) SPIRE 250, 350, and $500 \mu \mathrm{m}$ images. The PACS and SPIRE images are overlaid with the ${ }^{13}$ CO contours showing $75 \%$, $50 \%$, and $30 \%$ of the peak ${ }^{13} \mathrm{CO} J=2-1$ intensity $\left(T_{A}^{*}=2.9 \mathrm{~K}\right)$. The location of the central source is marked by a black circle in the ${ }^{13} \mathrm{CO}$ and $250 \mu \mathrm{m}$ images. Inset in panel (d) shows a 1.5 $\times 1.5$ region around the central source. A square-root stretch is used for the intensities in all panels except the inset, where a linear stretch is used. The angular resolutions (FWHM beam sizes) of the Herschel images at 70, 160, 250, 250, and $500 \mu \mathrm{m}$, respectively, are 8.4, 13.5, 18.2, 24.9, and 36.3 arcsec, and the peak intensities of the source are 517.6, 126.5 43.6, 12.9, and $3.1 \mathrm{mJy} \mathrm{arcsec}^{-2}$.

and $\mathrm{SiO}$ lines. This difference in profile asymmetries between the MVO and HVO strengthens our inference that these two outflows are distinct kinematic components.

The SO $N, J=5,6-4,5$ flux measured with the SMA is consistent with that measured with the SMT. We infer that the emission seen in the single-dish spectra of the SO arises in the pseudo-disk and MVO.

\subsubsection{Continuum}

The $1.3 \mathrm{~mm}$ continuum integrated over line-free channels, shows a largely unresolved source, located at coordinates (J2000 R.A., decl.): 5:53:43.570 24:14:44.47 (Figure 15(a)), with a FWHM size $\left(11^{\prime \prime} 7 \times 11^{\prime \prime} 1\right)$, and PA $\left(\sim 73^{\circ}\right)$ that is very similar to that of the beam $\left(1{ }^{\prime \prime} 6 \times 1{ }^{\prime \prime} 0, \mathrm{PA}=73^{\circ}\right)$. Hence, the intrinsic source size is $\lesssim 00^{\prime \prime} 47$. The $2.6 \mathrm{~mm}$ continuum source is also unresolved (Figure 15(b)) (beam 3." $0 \times 2$ !" 8 ).

The total flux at $1.3 \mathrm{~mm}$ is $\sim 50 \mathrm{mJy}(45 \mathrm{mJy}$ at a frequency of $220.4 \mathrm{GHz}$ from the lower sideband, and $56 \mathrm{mJy}$ at a frequency of $230.5 \mathrm{GHz}$ from the upper sideband). Comparing this with the continuum flux at $2.6 \mathrm{~mm}$ of $5 \pm 1 \mathrm{mJy}$ measured by Setal08, we derive a power-law dust emissivity exponent of 1.4 in the Rayleigh-Jeans approximation.

\section{The Structure of IRAS 05506}

The structure of IRAS 05506 and its environment is complex. However, as a result of being able to map several different molecular lines at different angular resolutions, we are able to identify the major components and derive their physical properties. Many of the derived values are sensitive to the adopted distance, and we have modified the $2.8 \mathrm{kpc}$ distance to IRAS 05506 adopted in Setal08. We use $V_{\mathrm{lsr}}=6 \mathrm{~km} \mathrm{~s}^{-1}$ to determine a distance of $3.7 \mathrm{kpc}$. This value is similar to that derived by Lumsden et al. (2013) as part of the Red MSX Source Survey of massive protostars.

Note, however, because of the typical intrinsic velocity dispersion in molecular clouds, the uncertainty in the distance to IRAS 05506 is relatively large. We show below that proper motion measurements of IRAS 05506's high-velocity bullets can be used to determine its expansion-parallax distance, with each bullet providing an independent measure of the distance.

The expected proper motion of the knots is $22.7 \mathrm{mas} \mathrm{yr}^{-1}$ $\sin i\left(V_{k} / 400 \mathrm{~km} \mathrm{~s}^{-1}\right)(3.7 \mathrm{kpc} / \mathrm{D})$, where $V_{k}$ is the $3 \mathrm{D}$ velocity of the knot and $i$ is the inclination of the axis, relative to the line of sight, along which each knot is moving. Both $V_{k}$ and $i$ can be estimated from the radial velocity profiles of the bow-shock emission associated with each knot. Bow-shock models show that for $i=90^{\circ}$, the velocity structure is symmetric around the systemic velocity and becomes increasingly asymmetric as $i$ becomes smaller or larger than $90^{\circ}$ (see Figure 1 of Hartigan et al. 1987: HRH87); the velocity width of the profiles at the base is equal to the 3D velocity of the shocked material at the apex of the bow-shock, i.e., $V_{k}$. We have extracted the $\mathrm{H} \alpha$ velocity profile of knot K1 in IRAS 05506's bullet spray from the optical spectroscopy in Setal08. The observed line profile, with a narrow peak near the systemic velocity and a broad 

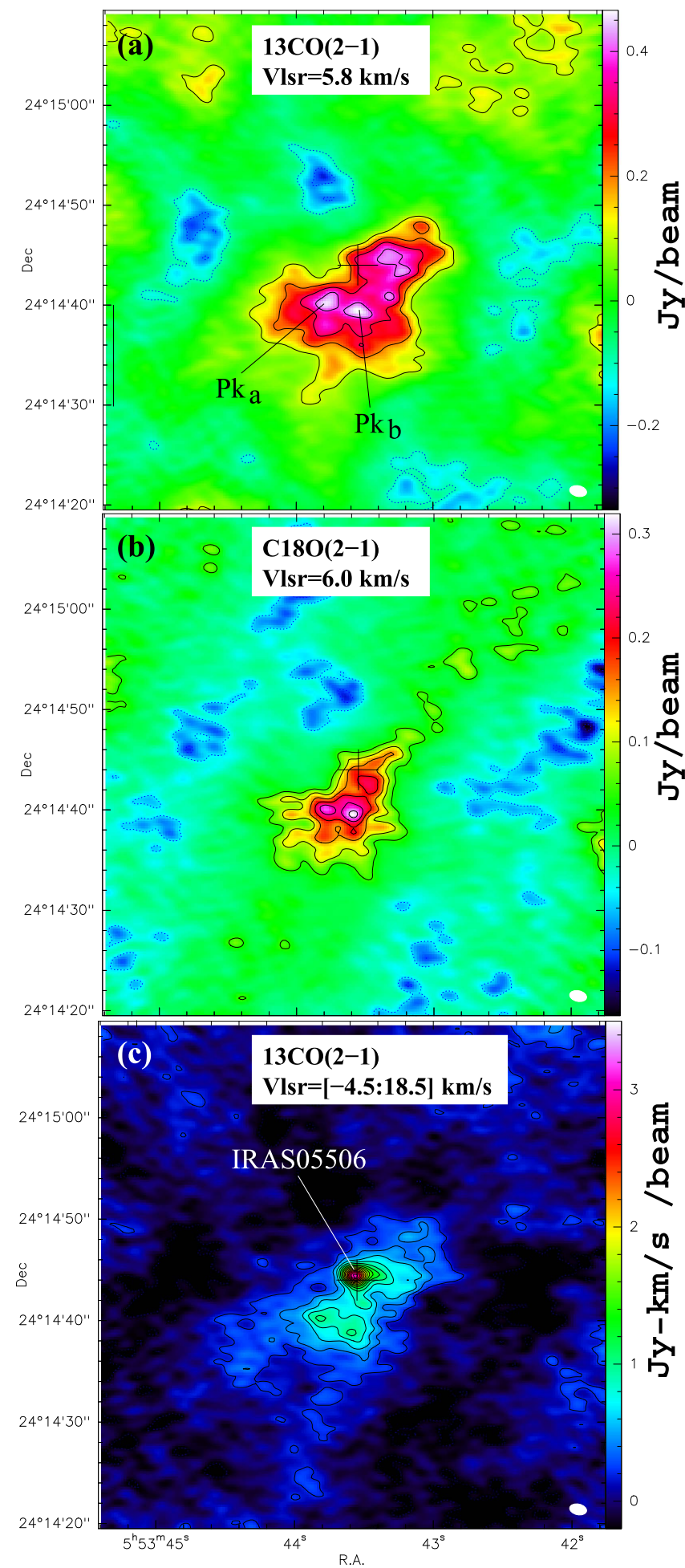

Figure 7. SMA map of IRAS 05506 Cloud B (a) in ${ }^{13} \mathrm{CO} J=2-1$ emission at $V_{1 \mathrm{sr}}=5.9 \mathrm{~km} \mathrm{~s}^{-1}$ (channel width is $1 \mathrm{~km} \mathrm{~s}^{-1}$ ); (b) $\mathrm{C}^{18} \mathrm{O} \mathrm{J}=2-1$ emission at $V_{\mathrm{lsr}}=6 \mathrm{~km} \mathrm{~s}^{-1}$ (channel width is $1 \mathrm{~km} \mathrm{~s}^{-1}$ ); and (c) in ${ }^{13} \mathrm{CO} J=2-1$ emission in the range $V_{\mathrm{lsr}}=-4.5$ to $18.5 \mathrm{~km} \mathrm{~s}^{-1}$. The beam FWHM is $1^{\prime \prime} 7 \times 1$." $05, \mathrm{PA}=78^{\circ} .1$ (white ellipses in lower-right corners of panels). Scale bar shows intensity units in Jy beam ${ }^{-1}$. The cross shows the location of the phase-center for the maps (J2000 R.A., decl.) 05:53:43.55, 24:14:44.0.

shoulder extending toward blueshifted velocities (Figure 16), appears to closely resemble the $i=60^{\circ}$ model profile for a shock velocity $V_{s}=400 \mathrm{~km} \mathrm{~s}^{-1}$, including its width at the base, $\sim 400 \mathrm{~km} \mathrm{~s}^{-1}$ (panel $\mathrm{N}$ in Figure 1 of HRH87). ${ }^{14} \mathrm{We}$ conclude that $i \sim 60^{\circ}$ for knot $\mathrm{K} 1$, and that $V_{k}=V_{s}=$ $400 \mathrm{~km} \mathrm{~s}^{-1}$. Thus, the total proper motion from our first-epoch imaging in 2002 October to (say) a second epoch in 2018, is 363 mas (3.7 kpc/D), and thus easily measurable, even for D as large as $\sim 15 \mathrm{kpc}$. Conservatively accounting for uncertainties in $i$ and the proper motion, the expansion-parallax distance to IRAS 05506 can be determined with an uncertainty of about $\pm 10 \%$.

The major components of IRAS 05506 and its environment are shown schematically in Figure 17 and described below. Some salient properties of these components are tabulated in Tables 3 and 4 for easy reference.

\subsection{Large-scale Structure}

\subsubsection{Cloud A and its Dense Core}

Our single-dish ${ }^{12} \mathrm{CO} J=2-1$ (e.g., Figure 2) and $J=1-0$ (Setal08) maps reveal that IRAS 05506 is located toward a very extended cloud (cloud A) with $V_{\mathrm{lsr}}=6 \mathrm{~km} \mathrm{~s}^{-1}$. The "absorption" feature that is seen in the ${ }^{12} \mathrm{CO} J=2-1$ and $1-0$ singledish spectra of IRAS 05506 at $V_{\mathrm{lsr}}=6 \mathrm{~km} \mathrm{~s}^{-1}$ is presumably due to relatively cold gas in this cloud that is optically thick in these lines. The brightness temperatures of the line emissions in these spectra at $V_{\mathrm{lsr}}=6 \mathrm{~km} \mathrm{~s}^{-1}(4 \mathrm{~K}$ and $2.5 \mathrm{~K}$, respectively, after convolving the ${ }^{12} \mathrm{CO} J=2-1$ data to the larger beam size of the ${ }^{12} \mathrm{CO} J=1-0$ data) indicate an average kinetic temperature $7 \mathrm{~K}$ in Cloud $\mathrm{A}$.

As noted previously (Section $3.1 .1,3.1 .3$ ), the ${ }^{13} \mathrm{CO} J=2-1$ map delineates a dense core (see Figures 3,6) within cloud A. We make the reasonable assumption that the single-dish profiles of ${ }^{13} \mathrm{CO}(J=1-0$ and $2-1)$ and $\mathrm{C}^{18} \mathrm{O}(J=1-0)$, all of which are centrally peaked at $V_{\mathrm{lsr}}=6 \mathrm{~km} \mathrm{~s}^{-1}$, arise from this dense core. The ratio of the single-dish ${ }^{13} \mathrm{CO} J=1-0$ to $\mathrm{C}^{18} \mathrm{O}$ $J=1-0$ spectra is 6.3 , close to the local interstellar ${ }^{13} \mathrm{CO}$ to $\mathrm{C}^{18} \mathrm{O}$ abundance ratio of $\left[{ }^{13} \mathrm{CO}\right] /\left[\mathrm{C}^{18} \mathrm{O}\right]=6.1$ (Wilson 1999), from which we infer that the ${ }^{13} \mathrm{CO} J=1-0$ line in the cloud A core, averaged over a $1^{\prime}$ scale, has an optical depth of slightly less than unity $(\sim 0.7)$, and the kinetic temperature is about $9.5 \mathrm{~K}$. The beam-averaged column density of $\mathrm{C}^{18} \mathrm{O}$ is $\mathrm{N}\left(\mathrm{C}^{18} \mathrm{O}\right)=6 \times 10^{14} \mathrm{~cm}^{-2}$, implying a molecular hydrogen column density, $N\left(\mathrm{H}_{2}\right)=3.5 \times 10^{21} \mathrm{~cm}^{-2}$, assuming a standard ISM $\mathrm{C}^{18} \mathrm{O} / \mathrm{H}_{2}$ abundance ratio of $1.7 \times 10^{-7}$ (Goldsmith et al. 1997).

\subsubsection{Cloud B}

Our SMA ${ }^{13} \mathrm{CO} J=2-1$ map resolves the smooth extended emission from cloud $\mathrm{A}$ and its core, and shows a roughly trapezoidal-shaped cloud (cloud B), extending about 14" (at its half-intensity contour) along $\mathrm{PA} \sim 135^{\circ}$ (Figure 7(a)), i.e., roughly along the axis of the wide-angle bullet spray. The $\mathrm{C}^{18} \mathrm{O}$ $J=2-1$ map shows a roughly similar shape (Figure 7(b)). A moment 0 map of the ${ }^{13} \mathrm{CO} J=2-1$ emission over the velocity range $V_{\mathrm{lsr}}=-4.5$ to $18.5 \mathrm{~km} \mathrm{~s}^{-1}$ shows the outflow source in IRAS 05506 located near the NE periphery of this cloud. Emission from cloud $\mathrm{B}$ is seen in the $V_{\mathrm{lsr}}=5-7 \mathrm{~km} \mathrm{~s}^{-1}$ range, peaking between channels centered at $V_{\mathrm{lsr}}=6$ and $V_{\mathrm{lsr}}=7 \mathrm{~km} \mathrm{~s}^{-1}$.

Assuming that the densities are high enough for the lines to be thermalized and $\left[{ }^{13} \mathrm{CO}\right] /\left[\mathrm{C}^{18} \mathrm{O}\right]=6.1$ (as above), we find

\footnotetext{
14 Although the profile shape in panel $\mathrm{R}$ also looks similar, its width at the base $\left(\lesssim 300 \mathrm{~km} \mathrm{~s}^{-1}\right)$ is significantly smaller than observed.
} 


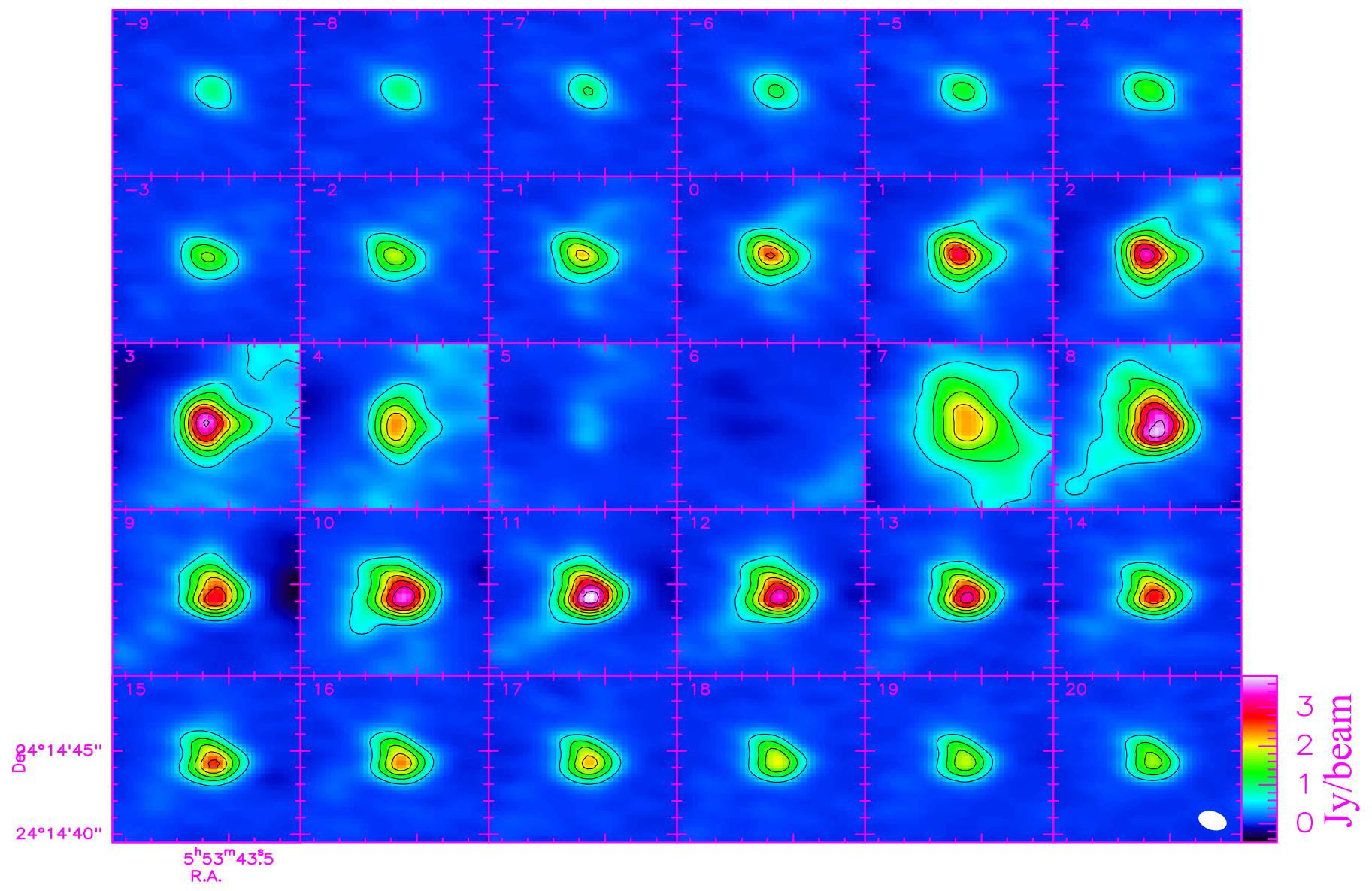

Figure 8. SMA ${ }^{12} \mathrm{CO} J=2-1$ channel map of IRAS 05506 covering a $10^{\prime \prime} \times 10^{\prime \prime}$ field-of-view and velocity range $V_{\text {lsr }}=-9$ to $20 \mathrm{~km} \mathrm{~s}^{-1}$. The beam FWHM is $1 . \prime 6 \times 1 . \prime 0, \mathrm{PA}=72^{\circ} .9$ (white ellipse in bottom-right panel).

that the ${ }^{13} \mathrm{CO} J=2-1$ emission is optically thick everywhere in cloud $\mathrm{B}$ by fitting the brightness temperatures of the ${ }^{13} \mathrm{CO}$ to $\mathrm{C}^{18} \mathrm{O} J=2-1$ emission in the SMA maps. Toward the location of IRAS 05506, the ${ }^{13} \mathrm{CO} J=2-1$ optical depth is $\tau_{13 \mathrm{CO} 21}=4$ and the kinetic temperature is $T_{\text {kin }}=8.5 \mathrm{~K}$. In comparison, $\tau_{13 \mathrm{CO} 21}=7$ and $T_{\text {kin }}=11 \mathrm{~K}$ in $\mathrm{Pk}_{b}$, located 5".3 south of IRAS 05506.

\subsubsection{Cloudlet B1}

The nature and association of Cloudlet B1 with the IRAS 05506 outflow source is not clear. For now, all we can say is that it produces additional features in some of the maps and spectra, adding to their complexity. For example, it is likely responsible for the emission peak near $V_{\mathrm{lsr}}=7.5 \mathrm{~km} \mathrm{~s}^{-1}$ seen in the single-dish profiles of ${ }^{12} \mathrm{CO} J=3-2$ and 4-3, and the prominent peak at $V_{\mathrm{lsr}}=7-8 \mathrm{~km} \mathrm{~s}^{-1}$ in the ${ }^{12} \mathrm{CO} J=2-1 \mathrm{SMA}$ extsub line profiles. This component was shown to be modestly extended, relative to the SMA extsub beam, but because the ratio of the ${ }^{12} \mathrm{CO} J=4-3$ to the $J=3-2$ peak intensity is about 1.8 , it must be significantly smaller than the ${ }^{12} \mathrm{CO} J=4-3$ beam size $\left(15^{\prime \prime}\right)$, in order for beam-dilution to account for this ratio.

\subsection{4. $S E O$}

The SEO extends over about $1 \mathrm{pc}$ and is seen in our single-dish ${ }^{12} \mathrm{CO} J=2-1$ maps. The age of the SEO, estimated by dividing the separation between its blue and redshifted lobes, of $\delta_{\mathrm{SEO}}=51^{\prime \prime} 8$ (as seen in the $V_{\mathrm{lsr}}$ velocity ranges $(0,4) \mathrm{km} \mathrm{s}^{-1}$ and $(8,12) \mathrm{km} \mathrm{s}^{-1}$ (Figure 4)), by the average velocity separation of $8 \mathrm{~km} \mathrm{~s}^{-1}$ between these lobes, is $t_{\text {SEO }} \sim 114,000$ year.

We estimate a total ${ }^{12} \mathrm{CO} J=2-1$ flux of 22.9 and 28.8 $\mathrm{Jy}-\mathrm{km} \mathrm{s}^{-1}$ in the blue and red lobes of the SEO, which, assuming a nominal kinetic temperature of $15 \mathrm{~K}$ and optically thin emission, implies a total mass, scalar momentum, and kinetic energy of $1.6 M_{\odot}, 4.7 M_{\odot} \mathrm{km} \mathrm{s}^{-1}$, and $0.3 \times 10^{45} \mathrm{erg}$, respectively. We have assumed a fractional $\mathrm{CO}$ abundance $f_{\mathrm{CO}}=[\mathrm{CO}] /\left[\mathrm{H}_{2}\right]=10^{-4}$ (here and elsewhere in the paper). Its presence implies that a very significant amount of kinetic energy has been added to a $\sim 1 \mathrm{pc}$ region around IRAS 05506 .

The sense of the velocity gradient, the axis orientation, and the scalar momentum, of the HVO and SEO are similar. Hence, it is likely that the SEO has resulted from the interaction of the HVO with the surrounding ambient cloud material. Thus, the value of $t_{\mathrm{SEO}}$ derived above is an upper limit. A better approximation of the SEO age can be derived assuming a uniform deceleration, in which case $t_{\mathrm{SEO}}=0.5 \delta_{\mathrm{SEO}} /\left(V_{i}+V_{f}\right)=9300$ year, where $V_{i}$ and $V_{f}$ are the initial and final velocities of the material in the SEO, taken to be the average outflow velocity of the HVO $\left(94 \mathrm{~km} \mathrm{~s}^{-1}\right.$; see Section 4.2 .2 below) and the SEO $\left(4 \mathrm{~km} \mathrm{~s}^{-1}\right)$.

\subsection{Small-scale Structure}

The central compact source in IRAS 05506, as seen in our high angular resolution molecular-line maps, has several major structural components that can be identified by their spectral signatures (Figure 10) and spatial extents; we describe these below. 


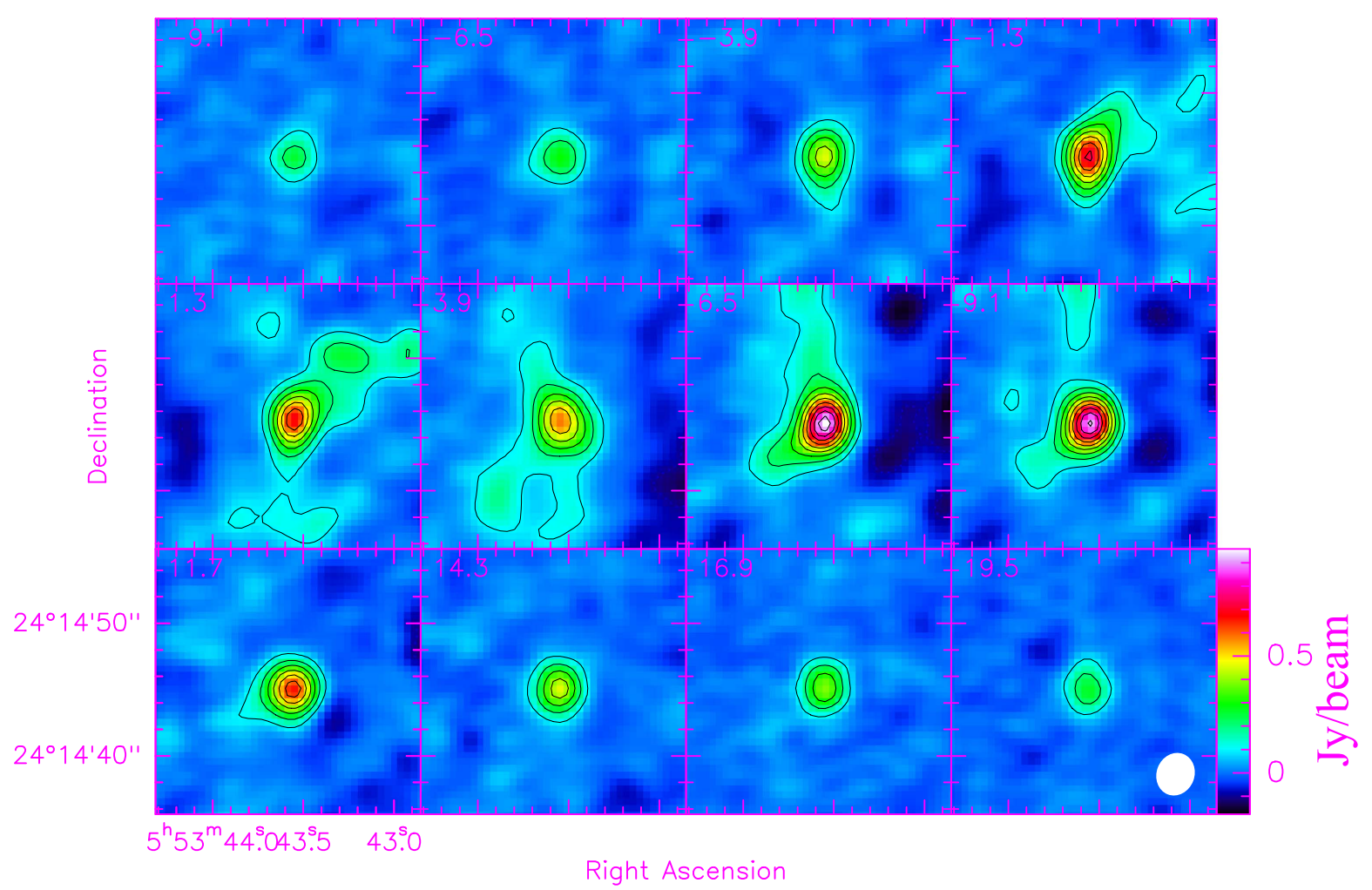

Figure 9. OVRO ${ }^{12} \mathrm{CO} J=1-0$ channel map of emission from IRAS 05506 covering a $20^{\prime \prime} \times 20^{\prime \prime}$ field-of-view and velocity range $V_{\mathrm{lsr}}=-9.1$ to $29.9 \mathrm{~km} \mathrm{~s} \mathrm{k}^{-1}$. The beam FWHM is 3 ". $1 \times 2$ " 7 , PA $=-21^{\circ}$ (white ellipse in bottom-right panel).

\subsubsection{The Pseudo-disk}

The pseudo-disk is seen in ${ }^{12} \mathrm{CO} J=2-1$ emission at $2.7<V_{\mathrm{lsr}}<10.9 \mathrm{~km} \mathrm{~s}^{-1}$, with its axis aligned roughly along that of the wide-angle bullet spray. We estimate a rough upper limit to the optical depth of the pseudo-disk emission at the velocity of the blue peak by comparing the average intensities of the ${ }^{12} \mathrm{CO} J=1-0(\sim 10 \mathrm{~K})$ and $J=2-1$ emission $(\sim 20 \mathrm{~K})$ in spectra extracted by averaging over a $3^{\prime \prime} \times 3^{\prime \prime}$ region, after convolving the ${ }^{12} \mathrm{CO} J=2-1$ map to the same beam size as the $J=1-0$ one. We find that the ${ }^{12} \mathrm{CO} J=2-1$ optical depth is $\sim 1$, implying an optical depth for ${ }^{13} \mathrm{CO} J=2-1$ that is a factor of 60 lower.

We have made a simple, squat-cylindrical, edge-on model of the pseudodisk, assuming that it has both a free-fall velocity (i.e., radial) component and rotation, with a conservation of angular momentum, using a code described in Lee et al. (2014). We have therefore assumed that $V_{\phi}(r)=V_{0, \phi}\left(r / 1^{\prime \prime}\right)^{-1}$ and $V_{r}(r)=-V_{0, r}\left(r / 1^{\prime \prime}\right)^{-0.5}$, and adjusted $V_{0, \phi}$ and $V_{0, r}$, in order to obtain a good fit to the contours in the PV plot shown in Figure 12, as well as the observed brightness. The temperature is assumed to be $T_{\mathrm{exc}}=T_{1}\left(r / 1^{\prime \prime}\right)^{-0.4}$, and the molecular hydrogen density, $\rho=\rho_{1}\left(r / 1^{\prime \prime}\right)^{-0.5}$. The temperature powerlaw index, -0.4 , is assumed to be the same as in low-mass envelopes (e.g., Hogerheijde 2001; Lee et al. 2006). The density power-law exponent, -0.5 , results in a constant mass infall rate, given our assumption of a fixed disk thickness and -0.5 for the power-law exponent of the infall velocity above. The parameters $T_{1}$ and $\rho_{1}$ are adjusted to reproduce the absolute intensity of the ${ }^{12} \mathrm{CO} J=2-1$ emission from the pseudo-disk. All models have a disk thickness of $2^{\prime \prime}$ and an outer disk radius of 1 "! $3-1$ "! 5 (the inner radius is assumed to be much smaller than the outer radius.) The model PV plot is generated after convolving the model brightness distribution with the beam.

We find good fits with $V_{0, r}=2-3 \mathrm{~km} \mathrm{~s}^{-1}, V_{0, \phi}=4 \mathrm{~km} \mathrm{~s}^{-1}$, $\rho_{1}=2.5 \times 10^{5} \mathrm{~cm}^{-3}$, and $T_{1}=65-75 \mathrm{~K}$. A representative model is shown in Figure 12. The central stellar mass is $M_{*}=(8.3-18.8)(D / 3.7 \mathrm{kpc}) M_{\odot}$ and the disk mass is $M($ disk, tot $)=(0.64-0.85)(D / 3.7 \mathrm{kpc})^{2} M_{\odot}$. This mass range is consistent with the mass of the young star associated with IRAS 05506, Sa, derived from SED modeling (Setal08: $11.2 M_{\odot}$ at $D=3 \mathrm{kpc}$ ), and scaled to our distance of $3.7 \mathrm{kpc}$.

The compact continuum source reported above (Section 3.2.3), is likely to be thermal dust emission from the dense inner region of the pseudo-disk. Without a detailed knowledge of the dust density structure, it is not possible to build a robust model of the thermal dust emission, although we can derive some rough constraints from the observed size of the continuum source. The characteristic dust emission radius, assuming no extinction or reddening of the starlight, is given by $r_{d}=\left[\frac{L_{*} T_{*}^{\beta}}{16 \pi \sigma}\right]^{1 / 2} T_{d}^{-(2+\beta / 2)}$, where $\beta$ is the dust emissivity exponent (Sahai et al. 1999). Given that $r_{d} \lesssim 0$ "'47, we then have $T_{d} \gtrsim 185 \mathrm{~K}$. We have taken $T_{*}=4700 \mathrm{~K}$ and a luminosity scaled up to $D=3.7 \mathrm{kpc}, L_{*}=8900 L_{\odot}$, from Setal08.

However, it is likely that there is significant absorption of the starlight by intervening dust close to the star. If we assume that, say, $20 \%-50 \%$ of the starlight is re-radiated at an effective temperature of $T_{*}=650-1000 \mathrm{~K}$ by a compact source of optically thick dust surrounding the central star, then $T$ (disk) dust $\gtrsim(84-111) \mathrm{K}$ in order that $r_{d} \lesssim 0$ "' 47 .

From our measured $1.3 \mathrm{~mm}$ dust continuum flux, the above dust temperature estimate, and a dust emissivity (per unit dust 

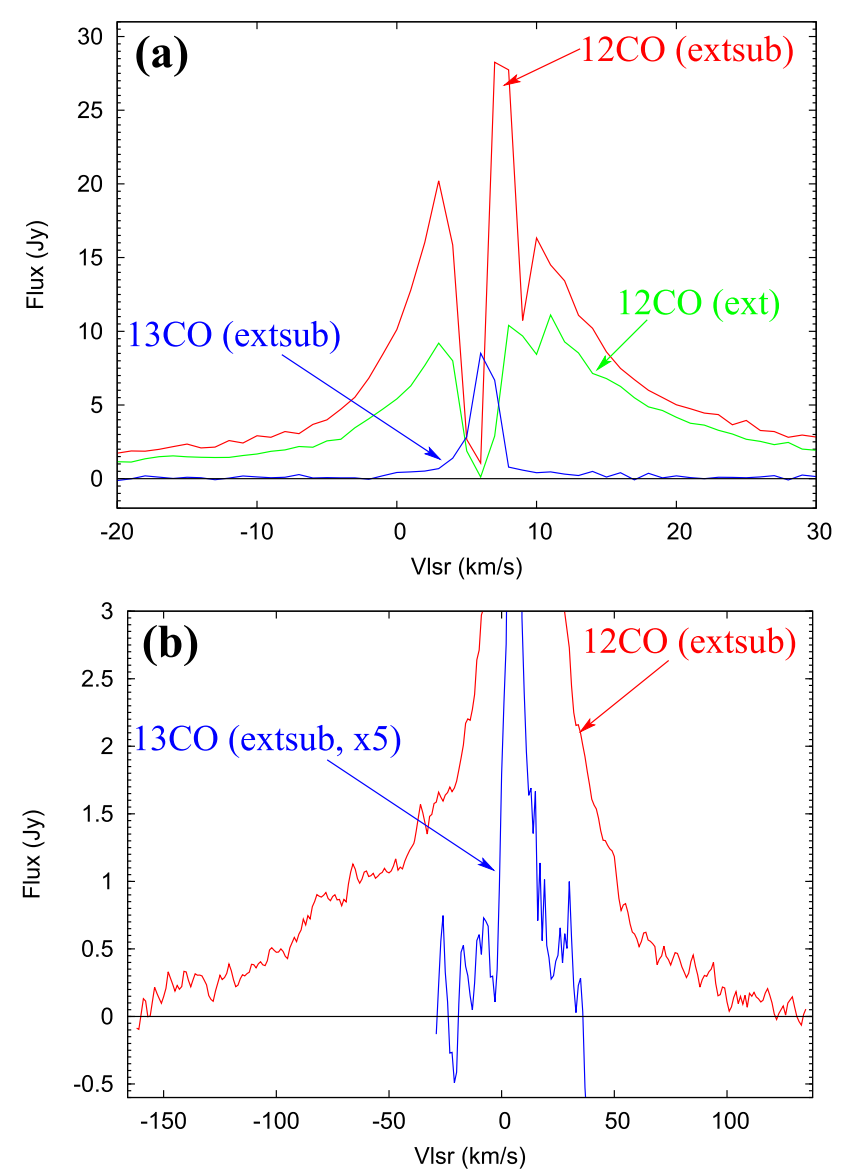

Figure 10. (a) Spatially integrated ${ }^{12} \mathrm{CO}$ and ${ }^{13} \mathrm{CO} J=2-1$ spectra extracted from a $10^{\prime \prime} \times 10^{\prime \prime}$ box centered on IRAS 05506, using the SMA data. For ${ }^{12} \mathrm{CO}$, the profiles have been extracted from the extsub (red) and ext (green) data cubes, and for ${ }^{13} \mathrm{CO}($ blue $)$, the profile has been extracted from the extsub array data cube. (b) The ${ }^{12} \mathrm{CO}$ and ${ }^{13} \mathrm{CO} J=2-1$ extsub profiles in panel (a) (smoothed using a three-point boxcar function), shown over a wider velocity range and a magnified intensity scale to show the faint high-velocity wings clearly (the ${ }^{13} \mathrm{CO}$ spectrum has been scaled up by a factor of five).

mass) of $\chi_{\nu}=1.5 \mathrm{~cm}^{2} \mathrm{~g}^{-1}$ at $1.3 \mathrm{~mm}$, we derive a dust mass and total mass of $M$ (disk, dust $) \lesssim(0.013-0.017) M_{\odot}$ and $M$ (disk, tot) $\lesssim(1.3-1.7) M_{\odot}$ (assuming the standard gas-todust ratio of 100). This disk mass is in reasonable agreement with that derived from our pseudo-disk kinematical model, given the uncertainties in the absolute value of the dust emissivity and our very simple thermal dust model.

\subsubsection{Outflows}

There are three outflows emanating from the location (or near vicinity of) IRAS $05506 \mathrm{Sa}$ - the SEO, HVO, and MVO. The physical properties of the SEO have been derived earlier (Section 4.1.4); those for the HVO and MVO are derived below.

The HVO: it is seen most clearly in ${ }^{12} \mathrm{CO} J=2-1$ emission at $V_{\text {lsr }} \lesssim-20 \mathrm{~km} \mathrm{~s}^{-1}$ and $V_{\mathrm{lsr}} \gtrsim 40 \mathrm{~km} \mathrm{~s}^{-1}$, and is aligned along PA $\sim 40^{\circ}$, with the blueshifted (redshifted) component $\mathrm{SW}$ (NE) of the center. The HVO axis is thus roughly orthogonal to that of the wide-angle spray $\left(121^{\circ}-155^{\circ}\right.$ : Setal08). This outflow was already noted by Setal08 in their ${ }^{12} \mathrm{CO}$ $J=1-0$ data, at a somewhat larger but uncertain PA, because the spatial separation of the blue- and redshifted components was small compared to the angular resolution of the data presented here $\left(3{ }^{\prime \prime} 9 \times 22^{\prime \prime} 9\right)$.

For this outflow, the ${ }^{12} \mathrm{CO} J=1-0$ and $J=2-1$ brightness temperatures in the HVO averaged over a $5.5^{\prime \prime}$ box, are rather low-e.g., about $0.4 \mathrm{~K}$ and $1 \mathrm{~K}$, respectively in the range $-50<V_{\text {lsr }}\left(\mathrm{km} \mathrm{s}^{-1}\right)<-20$, and about $0.1 \mathrm{~K}$ and $0.59 \mathrm{~K}$ in the range $40<V_{\mathrm{lsr}}\left(\mathrm{km} \mathrm{s}^{-1}\right)<70$. For larger velocity offsets from the systemic velocity, the brightness temperatures are even lower. The ratio of the $J=1-0$ to $2-1$ brightness temperature, $R_{12}$, is about 0.34 in the 40 to $110 \mathrm{~km} \mathrm{~s}^{-1}$ range (i.e., for the redshifted outflow), and 0.47 in the -20 to $-100 \mathrm{~km} \mathrm{~s}^{-1}$ range (i.e., for the blueshifted outflow), demonstrating that emission from both lines is optically thin.

The age of the HVO, estimated by dividing the separation of 1." 4 between blue- and redshifted lobes of the HVO, as seen in the velocity ranges $(-116,-84) \mathrm{km} \mathrm{s}^{-1}$ and $(72,104)$ $\mathrm{km} \mathrm{s}^{-1}$ (Figure 11), by the average velocity separation of $188 \mathrm{~km} \mathrm{~s}^{-1}$ between these lobes, is $t_{\mathrm{HVO}} \sim 130$ year.

The total ${ }^{12} \mathrm{CO} J=1-0$ and $2-1$ flux from the high-velocity blueshifted (redshifted) outflow component is 9.8 (2.5) and 80 (30) Jy $\mathrm{km} \mathrm{s}^{-1}$, from which we estimate that $T_{\text {kin }} \sim 20(30) \mathrm{K}$, and the mass, scalar momentum, and kinetic energy are about $0.052(0.022) M_{\odot}, 7.2 \quad(2.6) M_{\odot} \mathrm{km} \mathrm{s}^{-1}$, and $6.1 \times 10^{45}$ $\left(1.7 \times 10^{45}\right) \mathrm{erg}$, giving total mass, scalar momentum, and kinetic energy for the HVO of $0.074 M_{\odot}, 9.8 M_{\odot} \mathrm{km} \mathrm{s}^{-1}$, and $7.8 \times 10^{45} \mathrm{erg}$, respectively.

The MVO: it is seen most prominently in emission in the extreme wings of the $\mathrm{SO} N, J=5,6-4,5$ and $\mathrm{SiO} J=5-4$ lines in the SMA data. This outflow is relatively compact, hence we have used the ext data cube for our subsequent analysis of this line.

We estimate the age of the MVO as follows. First, we average the moment 0 maps of the blue-wing (i.e., $\left.-20<V_{\mathrm{lsr}}\left(\mathrm{km} \mathrm{s}^{-1}\right)<-6\right)$ and red-wing (i.e., $20<V_{\mathrm{lsr}}$ $\left.\left(\mathrm{km} \mathrm{s}^{-1}\right)<40\right)$ emission, and determine its size to be 1 ." $6 \times 1$ "! 3 (FWHM). Deconvolving this with the beam, 1 ." $46 \times 0$ ". 85 , we obtain a deconvolved size of 0 ". $8 \times 1$." 0 . We then divide the mean size by the average velocity separation between the blue and red-velocity intervals, i.e., $43 \mathrm{~km} \mathrm{~s}^{-1}$, giving an age $t_{\mathrm{MVO}} \sim 360$ year. The (relatively uncertain) age of the MVO is thus comparable to that derived for the knots in the wide-angle spray by Setal08: e.g., 265(D/ $3.7 \mathrm{kpc})$ and $225(D / 3.7 \mathrm{kpc})$ for knots $\mathrm{K} 1$ and $\mathrm{K} 2$, suggesting that these outflows are associated with the same triggering event.

Convolving the ${ }^{12} \mathrm{CO} J=2-1$ extsub data cube to the same angular resolution as the ${ }^{12} \mathrm{CO} J=1-0$ data cube, we find that the flux ratio of the ${ }^{12} \mathrm{CO} J=1-0$ to $2-1$ in the MVO is about 0.1 for the blueshifted component and 0.075 for the redshifted component. Modeling these ratios shows that emission from both lines is optically thin, and that the kinetic temperature lies in the $20-30 \mathrm{~K}$ range. From the total ${ }^{12} \mathrm{CO} J=2-1$ flux of 8.3 (14.7) $\mathrm{Jy} \mathrm{km} \mathrm{s}^{-1}$ for the blueshifted (redshifted) component of the MVO extracted from an aperture equal to the FWHM size of the MVO, we estimate the mass, scalar momentum, and kinetic energy ${ }^{15}$ to be about $0.011\left(0.02 M_{\odot}, 0.21\right.$ $(0.44) M_{\odot} \mathrm{km} \mathrm{s}^{-1}, 0.78 \times 10^{44}\left(2.1 \times 10^{44}\right)$ erg. The total mass, scalar momentum, and kinetic energy in the MVO are thus $0.031 M_{\odot}, 0.65 M_{\odot} \mathrm{km} \mathrm{s}^{-1}$, and $2.9 \times 10^{44} \mathrm{erg}$, respectively.

\footnotetext{
15 Because the fractional flux within an aperture of size equal to the FWHM of a Gaussian distribution is 0.5 of the total.
} 


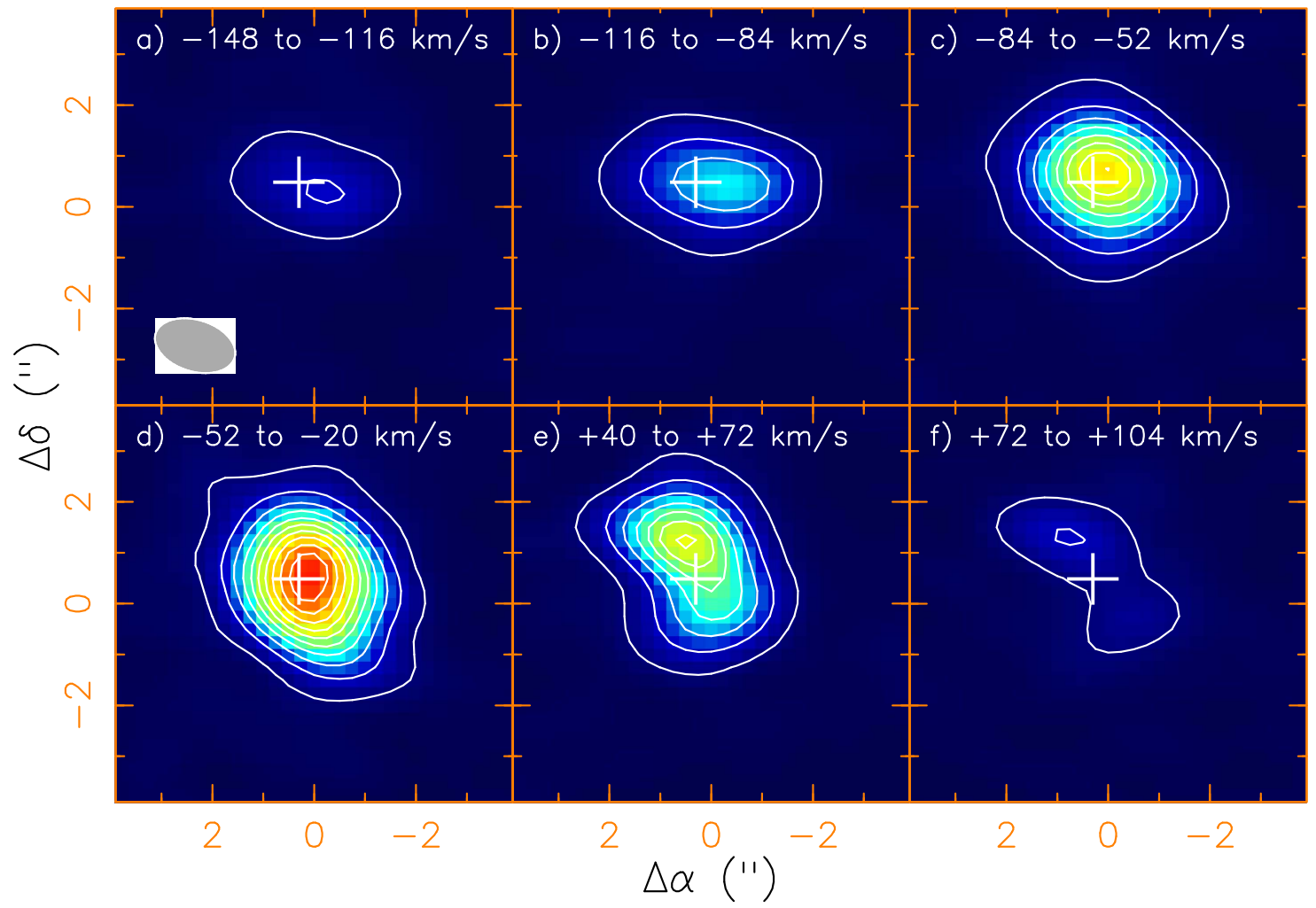

Figure 11. SMA map of the ${ }^{12} \mathrm{CO} J=2-1$ emission from IRAS 05506 integrated over the extreme blueshifted and redshifted velocity ranges. The cross shows the location of the optical/IR source Sa. Contour levels are $0.8+1.3 n \mathrm{Jy} \mathrm{beam}^{-1} \mathrm{~km} \mathrm{~s}^{-1}$, with $n=0,1,2, \ldots$ The beam is shown as a gray ellipse in panel (a).

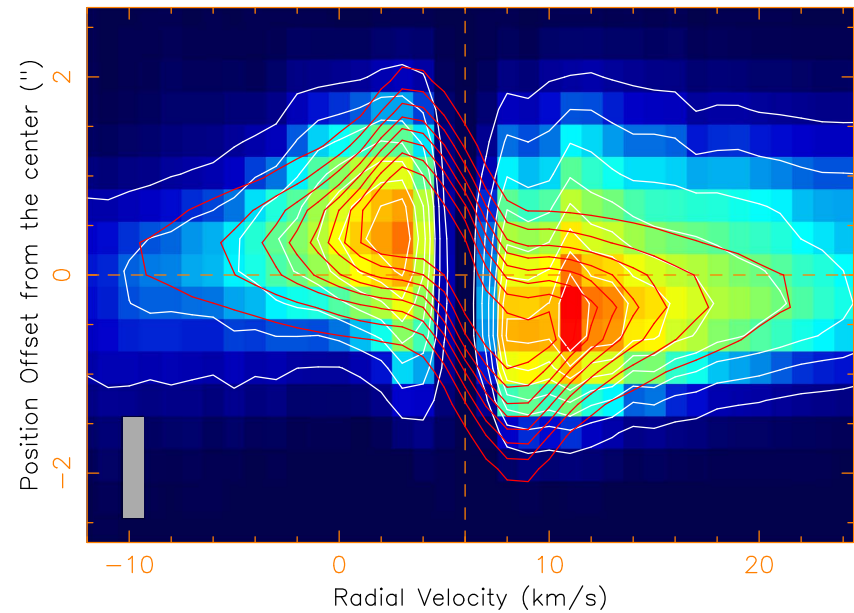

Figure 12. Observed and model position-velocity plots of the ${ }^{12} \mathrm{CO}$ intensity along a cut at $\mathrm{PA}=40^{\circ}$. Colorscale and white contours, with levels $0.3 n$ Jy beam ${ }^{-1}, n=1,2, \ldots$, show the observed intensity extracted from the SMA ${ }^{12} \mathrm{CO} J=2-1$ ext data cube. Negative (positive) position offsets are NE (SW) of center. The intensity from a representative model of an edge-on flat pseudodisk disk with infall and rotation (see text for further details), is shown in red contours, using the same intensity levels as for the data. A gray rectangle shows the velocity and angular resolution, and a dashed vertical line denotes the systemic velocity, $6 \mathrm{~km} \mathrm{~s}^{-1}$.

We can also use the SO data to further constrain the properties of the shocked gas in the MVO. The peak intensity of the SO $N, J=5,6-4,5$ source in the SMA extended array data is $1 \mathrm{Jy} \mathrm{beam}^{-1}$, implying a brightness temperature of $26 \mathrm{~K}$, and because the source is marginally resolved at best, this should be considered a lower limit.
Because the broad emission in the four SO lines observed with the ARO (Figure 5) likely arises in the MVO (Section 3.2.2), we can use the relative ratios of the integrated fluxes in the velocity ranges for the MVO component for these lines (corrected for beam-dilution) to constrain the density and temperature of shocked gas in the MVO. As these data have modest $\mathrm{S} / \mathrm{N}$, we only use the red component of the MVO, which appears to be stronger than the blue one, to compute these ratios. We find that the uncorrected ratios for the four SO lines 1, 2, 3, and 4 (Section 3.1.2), are 0.26:0.26:0.51:1. The ratios, corrected for beam-dilution, are 0.69:0.51:0.72:1, assuming the source-size is significantly smaller than the single-dish beams. We have used the non-LTE RADEX code (van der Tak et al. 2007) to compute the line ratios for a variety of kinetic temperatures and densities, and we find that only for densities $\rho\left(\mathrm{H}_{2}\right) \gtrsim 10^{7} \mathrm{~cm}^{-3}$ does the excitation temperature of the $5,6-4,5$ and $6,7-5,6$ lines approach the kinetic temperature; at this density, we find that $T_{\text {kin }} \gtrsim 80 \mathrm{~K}$ for a reasonable fit to the line ratios (for lower values of $T_{\text {kin }}$, the model intensities of the lower-frequency pair, $N$, $J=3,4-2,3$ and $N, J=4,3-3,2$, become too large relative to the higher frequency pair, $N, J=6,7-5,6$ and $N, J=5,6-4,5)$. Fits can be obtained at somewhat lower densities as well, by increasing the kinetic temperature - the lowest viable value of the density is $\rho\left(\mathrm{H}_{2}\right) \sim 10^{6} \mathrm{~cm}^{-3}$, for which the temperature is $T_{\text {kin }} \sim 120 \mathrm{~K}$. The significantly higher temperatures derived from the SO lines compared to the CO lines imply that the former is tracing the hot, shocked fraction of the material in the MVO.

\section{Discussion}

The closest counterpart to the wide-angle bullet spray that Setal08 found toward IRAS 05506 is the explosive BN-KL wide-angle outflow in OMC1. Furthermore, in IRAS 05506, 

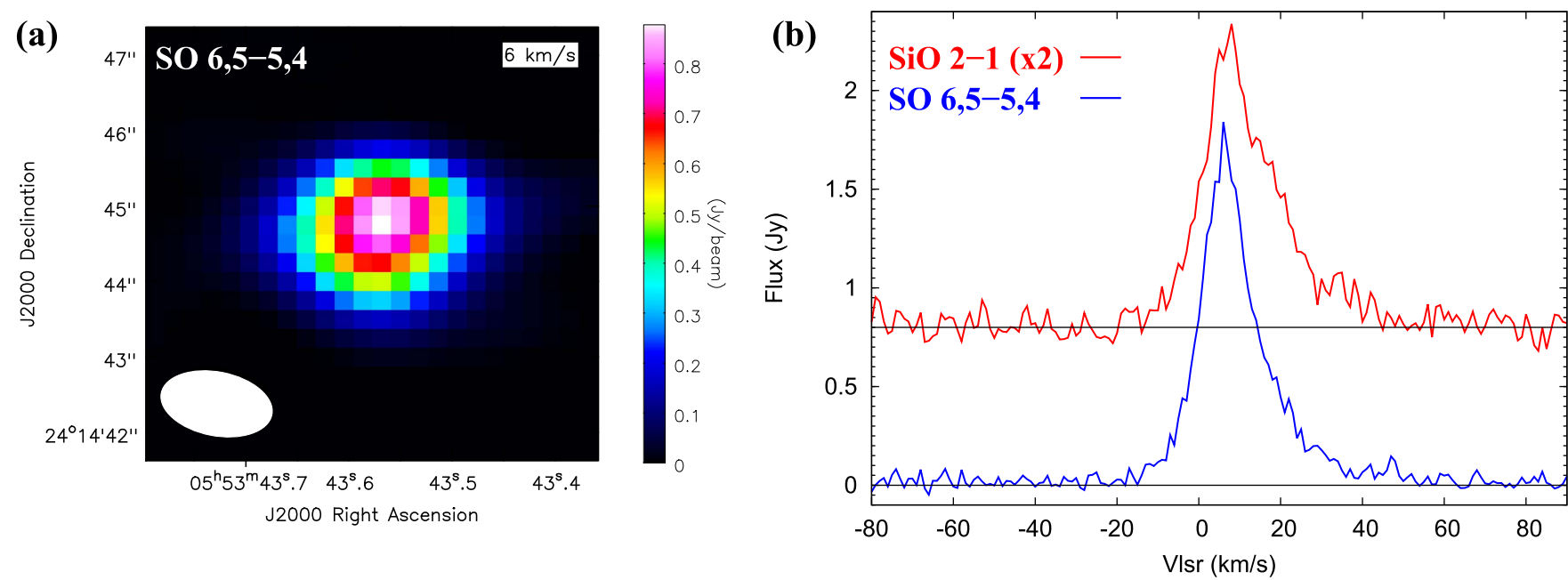

Figure 13. (a) SO $N, J=6,5-5,4$ map of IRAS 05506 obtained with the SMA (ext data cube) at the peak emission velocity, $V_{\mathrm{lsr}}=6 \mathrm{~km} \mathrm{~s}^{-1}$ (the beam FWHM is $1^{\prime \prime} .5 \times 00^{\prime \prime} 85, \mathrm{PA}=78.5$ (white ellipse). (b) $\mathrm{SO} N, J=6,5-5,4$ and $\mathrm{SiO} v=0, J=2-1$ spectra extracted from the ext data cube using apertures of size $3{ }^{\prime \prime} 6 \times 3{ }^{\prime \prime} 0$ and 3 " $9 \times 2$ " 9 , respectively (i.e., twice the FWHM source sizes in each line).

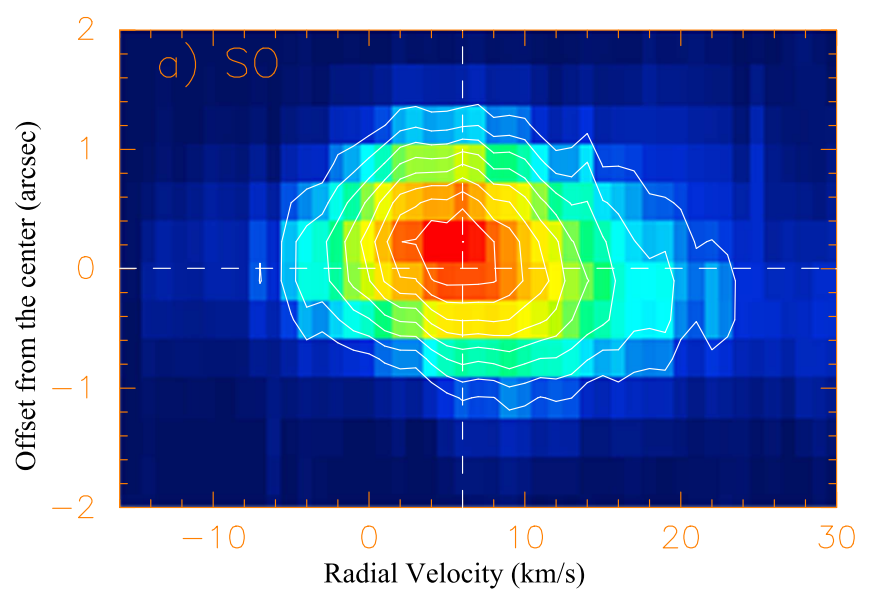

Figure 14. Position-velocity plot of the intensity along a cut at $\mathrm{PA}=40^{\circ}$ in the SO $N, J=5,6-4,5$ ext data cube. Minimum contour level (spacing) is 20 (10)\% of the peak intensity. Negative (positive) position offsets are NE (SW) of center.

the HVO is directed along an axis orthogonal to that of the bullet-spray, a likely counterpart to the collimated outflow that emerges from Source I in BN/KL (Plambeck et al. 2009; Zapata et al. 2012) along an axis that is orthogonal to the wideangle outflow as seen in $\mathrm{H}_{2}$ and [Fe II] line emission (Allen \& Burton 1993, BCM11). However, in addition to these similarities, we note that IRAS 05506 shows three additional components, namely the pseudo-disk, MVO and SEO, for which there are no clear counterparts in BN/KL.

The wide-angle bullet spray shares a common axis with the pseudo-disk and has an age comparable to that of MVO, suggesting that these three structures are intimately linked. The rotation of the pseudo-disk may be a result of the overall rotation of cloud $\mathrm{B}$, for the following reasons. The observed alignment of cloud B's long axis with that of the wide-angle outflow is unlikely to be due to the impact of the outflow on Cloud $\mathrm{B}$, as there is no obvious velocity gradient seen along this axis in cloud B. It is plausible that cloud B's long axis is aligned with its rotational axis, and thus cloud $\mathrm{B}$ is the preexisting dense cloud core in which IRAS 05506's pseudo-disk was formed. This would naturally account for these two structures having a common axis.

We note that Cloud B shows several distinct compact sources of dense gas (e.g., $P k_{a}, P k_{b}$; see Figure 7) in emission at the systemic velocity $V_{\mathrm{lsr}} \sim 6 \mathrm{~km} \mathrm{~s}^{-1}$, which do not appear to be associated with stars or outflows. In contrast, IRAS05506, which is associated with a massive star and multiple outflows, cannot be distinguished from Cloud B emission at the systemic velocity $V_{\mathrm{lsr}} \sim 6 \mathrm{~km} \mathrm{~s}^{-1}$. Thus, much of the low-velocity gas in the pre-existing density peak where IRAS05506's massive star was born has largely been removed, presumably by the outflows that came into existence after its formation.

We briefly discuss below past and current models for the $\mathrm{BN} / \mathrm{KL}$ outflows, in order to investigate their applicability to IRAS 05506. A model involving dynamical decay of a nonhierarchical system of massive stars was proposed for the BN/KL wide-angle outflow (Bally \& Zinnecker 2005; Rodríguez et al. 2005, BCM11), with the two most massive components of the stellar system being BN (10-13 $M_{\odot}$ runaway B star) and radio Source I (a hypothetical massive binary). BCM11 proposed that these stars were ejected from their natal cloud core due to a dynamical interaction between them, and that the gravitational energy released by the formation of binary Source I powers the bullet spray.

An alternative hypothesis was proposed by Tan (2004) and Chatterjee \& Tan (2012), in which the BN-KL outflow source is produced by the chance encounter of a runaway star $(\mathrm{BN})$ with the KL star-forming core. According to this hypothesis, BN was supposedly ejected from the $\theta^{1}$ Ori $C$ system 4500 year ago, and its interaction with the KL cloud within the last 1000 years has produced recently enhanced accretion and outflow activity (i.e., the bullet spray) from Source I.

The major weaknesses in each of the above models have been summarized in a recent study by Luhman et al. (2017). In the dynamical decay model, the mass of $\sim 20 M_{\odot}$ for Source I implied by conservation of momentum with $\mathrm{BN}$ alone is much larger than the dynamical mass of Source I ( $7 M_{\odot}$ : Plambeck \& Wright 2016). In the chance-encounter model, there are two problems-first, BN appears to be younger than the Trapezium stars, and second, this model does not explain the large motion of Source I. 

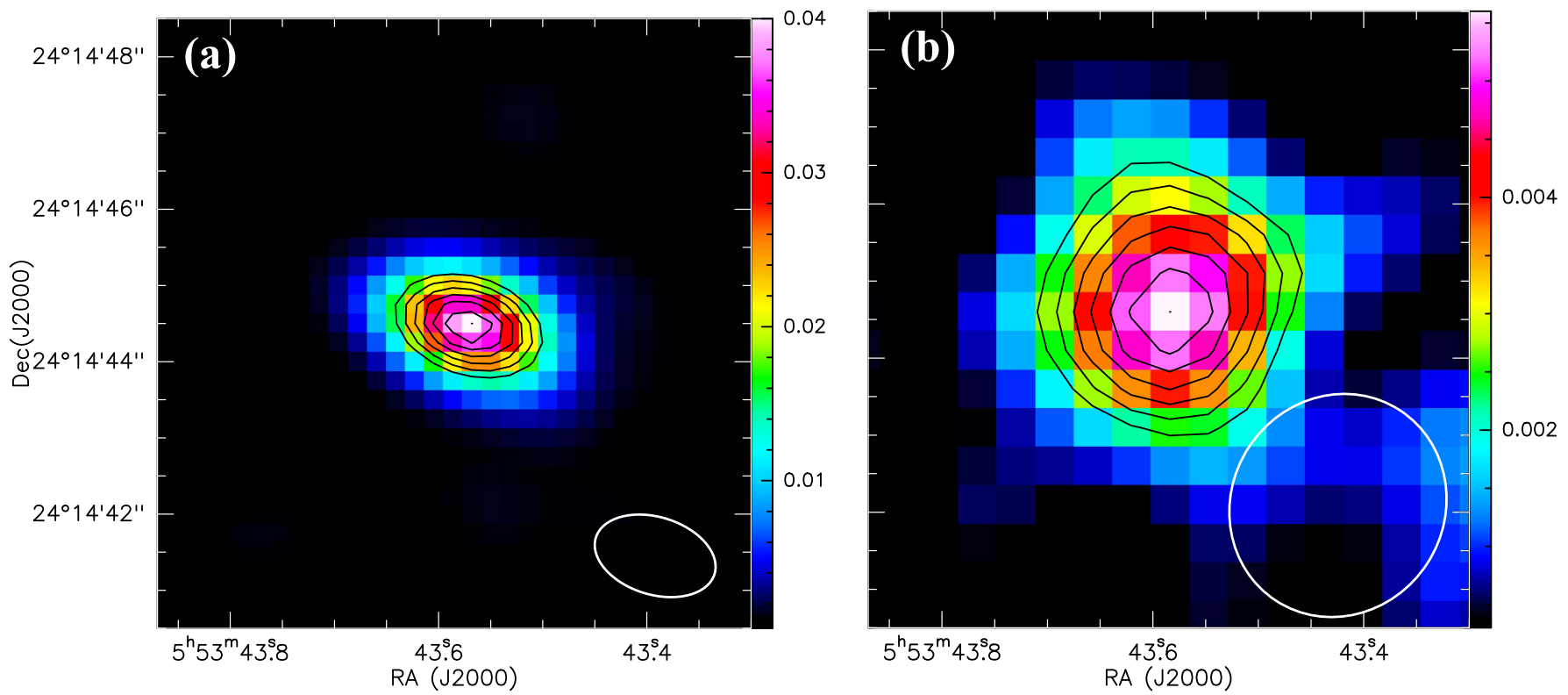

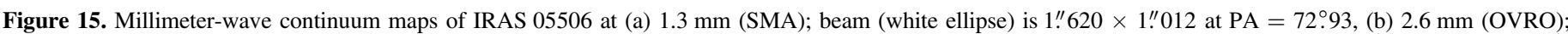

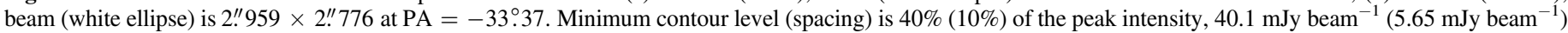
at $1.3(2.6) \mathrm{mm}$

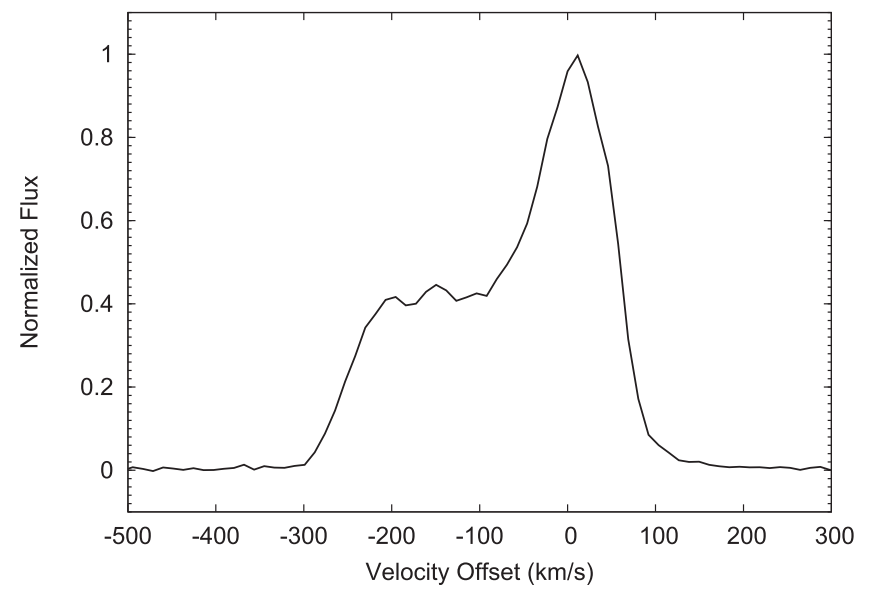

Figure 16. $\mathrm{H} \alpha$ velocity profile of knot K1 in IRAS 05506's bullet spray, from the optical spectroscopy in Setal08.

Goddi et al. (2011: Getal11) discuss the above models and two additional ones (numbered 1-4 in Section 6 of their paper). The latter involve a close passage between two (initially unbound) protostars, BN and Source I. In the first of these (no. 2), this passage produces enhanced accretion in the disk of Source I, but Goddi et al. reject this model because a binary encounter cannot produce stellar ejection, as appears to be the case for BN and Source I. In the second one (no. 4), favored by Getal11, Source I was originally a soft, massive $\left(20 M_{\odot}\right)$ binary that was hardened as a result of the encounter with $\mathrm{BN}$, and the resulting increase in gravitational potential energy powered both the wide-angle spray and the ejection of $\mathrm{BN}$ and Source I.

Luhman et al. (2017) provide new evidence that resolves the mass discrepancy for Source I mentioned above, thus strengthening the dynamical decay model as the likely one for the BN/KL explosive outflow. Specifically, they find that source $\mathrm{x}$ (from Lonsdale et al. 1982) in the KL nebula, which has a high proper motion, was involved in the dynamical decay that separated Source I from BN, and source X's momentum reduces the momentum requirement for Source I, bringing the latter's mass inferred from momentum balance into consistency with its dynamical mass. However, the relatively large value of the disk mass in Source $\mathrm{I}\left(\sim 0.1 M_{\odot}\right)$ is difficult to obtain in the dynamical decay model, either from retention of a pre-existing disk or reformation in the short time that has elapsed after the decay ( 500 year) (Plambeck \& Wright 2016).

Because we have not identified the multiple individual members of a dynamically decaying stellar cluster that may be associated with IRAS 05506, we cannot yet test the dynamical decay model for this source. We note, however, that near-IR $H S T$ imaging shows the presence of several faint stars (stars fs1, fs2, fs3, fs4 in Figure 2 of Setal08) and bright stars (e.g., $\mathrm{Sb}$; see Figures 2 and 5 of Setal08) within a $\sim 5^{\prime \prime}$ radius around the central optical/infrared source of IRAS 05506, Sa, some of which may be members of such a cluster. Second-epoch HST imaging observations can be used to look for proper motions of the stars found in the near vicinity of Sa. If any of the stars around the IRAS 05506 outflow source were ejected from a common center somewhere in the vicinity of IRAS $05506 \mathrm{Sa}$ as a result of the dynamical decay, then their typical angular offsets of about $2-5^{\prime \prime}$ from $\mathrm{Sa}$ let us estimate that the corresponding stellar proper motions would be in the range of $8-20 \mathrm{mas} \mathrm{yr}^{-1}$. The resulting total proper motion of 128-320 mas over 16 years between epoch 1 and a future observing epoch 2 is easily measurable.

The rather long age of the SEO may argue against the dynamical decay model of BCM11 for IRAS 05506. In this model, the collimated outflow from Source I results from a disk that is formed after the explosive disintegration event has produced the wide angle spray. ${ }^{16}$ Thus, the collimated outflow in Source I is younger than the wide angle spray, i.e.,

\footnotetext{
16 The apparent major axis of the disk in Source I (and in BN) is aligned with Source I's (BN's) proper motion; BCM11 explain this as the result of these disks being formed by Bondi-Hoyle accretion onto these stars from the cloud through which they are moving, assuming the latter has a significant density or velocity gradient orthogonal to the stellar motion (see the last paragraph in Section 4.2 of their paper).
} 


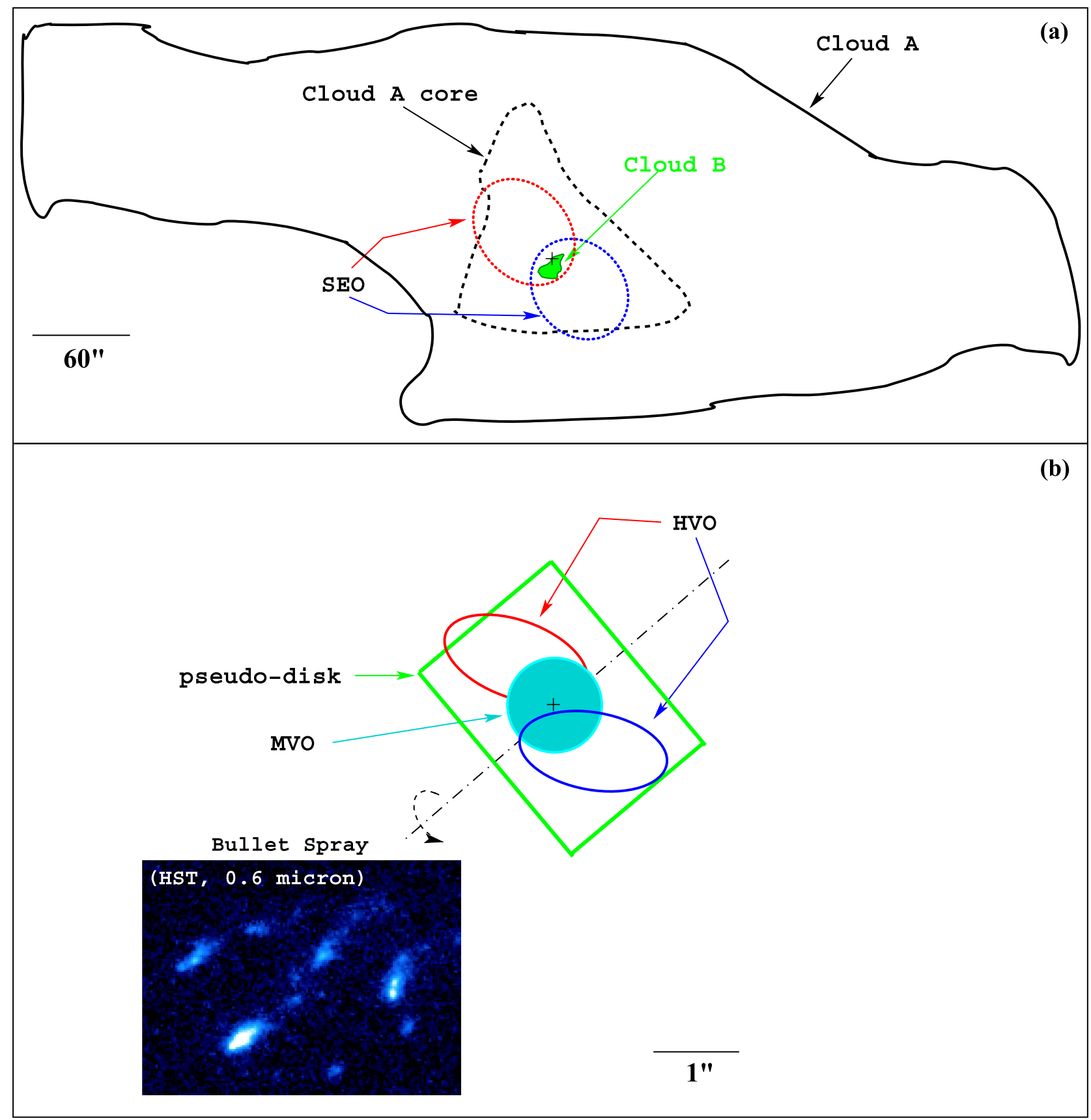

Figure 17. Schematic showing the various structural components of IRAS 05506 and its extended environment. The sizes of the various extended cloud components, the pseudo-disk, and the separations of red and blueshifted lobes of the HVO and SEO are roughly to scale. The sizes and shapes of the red- and blueshifted lobes of the SEO and HVO are only indicative. (a) The very extended cloud A, its dense core (cloud A-core), cloud B, and the slow extended outflow (SEO); the location of IRAS $05506 \mathrm{Sa}$ is marked with a cross. (b) The high-velocity outflow (HVO), the medium-velocity outflow (MVO), and the edge-on pseudo-disk (all of which lie inside cloud B), associated with Sa (black cross). Inset in panel (b) shows the high-velocity bullet spray found by Setal08 at the actual location relative to the central source. The rotation axis and sense of rotation of the pseudo-disk are shown by the black dash-dotted line and arrowed arc.

$\lesssim 500$ year. However the SEO's age of $\sim 9300$ years suggests that the HVO (the presumed counterpart to the collimated outflow from Source I), which has likely produced the SEO (Section 4.2.2), has been in operation for an equally long time. Thus, contrary to the expectation of the dynamical decay model, the HVO (and thus the disk that drives it) is much older than the bullet-spray in IRAS $05506 .{ }^{17}$ If the chance-encounter

\footnotetext{
17 An obvious caveat to this line of reasoning is that the disk in the IRAS 05506 source may be long-lived, having survived the violent disintegration process.
}

model does apply to IRAS 05506 , then the origin of the MVO and bullet spray is due to a sudden increase in the accretion rate of the pseudo-disk, resulting from a chance-encounter of a runaway star with the latter.

A model involving close passage between two protostars (no. 2 of Getal11) may also be applicable to IRAS 05506, because (unlike $\mathrm{BN} / \mathrm{KL}$ ) we do not have any evidence for a stellar ejection in its case. If so, then one of the two protostars in IRAS 05506 is the massive star associated with the pseudodisk (i.e., Sa), and the other is a star with a disk that produces the HVO. The observed specific orientations of the axes of the 
Table 3

Physical Structures Associated with IRAS05506: Observed Properties

\begin{tabular}{|c|c|c|c|c|c|}
\hline Name & $\begin{array}{c}V_{\text {lsr }} \text { Range } \\
\left(\mathrm{km} \mathrm{s}^{-1}\right)\end{array}$ & $\begin{array}{l}\text { Size } \\
\left({ }^{\prime \prime}\right)\end{array}$ & $\begin{array}{l}\text { PA } \\
\left({ }^{\circ}\right)\end{array}$ & Tracer & Data Set \\
\hline Central Star(s) & $\ldots$ & $\ldots$ & $\ldots$ & $\ldots$ & $\ldots$ \\
\hline Pseudo-disk & -6 to 20 & $2 \times 2.8^{\mathrm{a}}$ & $130^{\mathrm{b}}$ & $\mathrm{CO}(2-1)$ & SMA Ext \\
\hline HVO & -155 to -20 and 40 to 120 & 1.4 & $40^{\mathrm{b}}$ & $\mathrm{CO}(2-1)$ & SMA Ext + Sub \\
\hline Bullet Spray ${ }^{\mathrm{c}}$ & -300 to 100 & 5.3 & $120-155$ & $\mathrm{H} \alpha$ & $H S T / \mathrm{WFC}$ \\
\hline SEO & 0 to 5 and 7 to 12 & 52 & $45^{\mathrm{b}}$ & $\mathrm{CO}(2-1)$ & SMT \\
\hline Cloud A Core & 5 to 7 & $\sim 125$ & $45^{\mathrm{e}}$ & ${ }^{13} \mathrm{CO}(2-1), 250-500 \mu \mathrm{m}$ & SMT, HSO \\
\hline Cloud A & 5 to 7 & $>690(\mathrm{EW}) \times 310(\mathrm{NS})$ & $\sim 90^{\mathrm{c}}$ & ${ }^{12} \mathrm{CO}(2-1)$ & SMT \\
\hline$", "$ & 5 to 7 & $>1800(\mathrm{EW}) \times 600(\mathrm{NS})$ & $\ldots$ & ${ }^{12} \mathrm{CO}(1-0)$ & $\mathrm{KP} 12 \mathrm{~m}$ \\
\hline
\end{tabular}

Notes.

${ }^{a}$ (Vertical thickness) $\times$ (diameter).

${ }^{b}$ PA of symmetry axis.

${ }^{\mathrm{c}}$ Data from Sahai et al. (2008).

${ }^{\mathrm{d}} \mathrm{PA}$ of long axis.

${ }^{\mathrm{e}} \mathrm{PA}$ of hypotenuse, for this right-angled, triangular-shaped structure.

Table 4

Physical Structures Associated with IRAS05506: Derived Properties

\begin{tabular}{|c|c|c|c|c|}
\hline Name & $\begin{array}{l}\text { Age } \\
\text { (year) }\end{array}$ & $\begin{array}{l}\text { Mass } \\
\left(M_{\odot}\right)\end{array}$ & $\begin{array}{l}\text { Scal. Mom. } \\
\left(M_{\odot} \mathrm{km} \mathrm{s}^{-1}\right)\end{array}$ & $\begin{array}{c}\text { Energy } \\
\left(10^{45} \mathrm{erg}\right)\end{array}$ \\
\hline Central Star(s) & $\cdots$ & $8.3-18.8$ & $\ldots$ & $\cdots$ \\
\hline Pseudo-disk & $\cdots$ & $0.64-1.7$ & $\ldots$ & $\cdots$ \\
\hline MVO & 360 & 0.031 & 0.65 & 0.29 \\
\hline HVO & 130 & 0.074 & 9.8 & 7.8 \\
\hline Bullet Spray ${ }^{a}$ & 230 & $4.6 \times 10^{-5}$ & 0.018 & 0.074 \\
\hline SEO & 9300 & 1.6 & 4.7 & 0.3 \\
\hline
\end{tabular}

Note.

${ }^{\text {a }}$ For knot $K 1$, using data from Sahai et al. (2008), scaled to a distance $D=3.7 \mathrm{kpc}$.

pseudo-disk and the HVO (which are roughly orthogonal) would then be purely coincidental.

\section{Conclusions}

We have carried out a study of molecular-line and continuum emission from the interstellar bullet source IRAS 05506 and its environment, using both single-dish and interferometric observations. Our major conclusions are listed below:

1. The central optical/IR source Sa in IRAS 05506 is located at the northeast periphery of a dense, trapezoidshaped cloud (cloud B) with an extent of about 14" (at its half-intensity contour). Cloud B lies within the dense core (Cloud A core, size $\sim 2$ ! 1) of a large cloud (cloud A, size $11 ! 2 \times 5 ! 2$ ). The radial velocity of clouds $A$ and $B$ is $V_{\mathrm{lsr}} \sim 6 \mathrm{~km} \mathrm{~s}^{-1}$. The Cloud A dense core is prominently seen in thermal dust emission at wavelengths in the 250-500 $\mu \mathrm{m}$ range, as observed with the Herschel/ SPIRE camera.

2. A very extended bipolar outflow (SEO), of size $\sim 0$ ! 9 with its axis aligned along PA $\sim 45^{\circ}$, appears to emanate from the central source in IRAS 05506.

3. The central source consists of a pseudo-disk and two compact outflow components, a high-velocity outflow
(HVO), and a medium-velocity outflow (MVO). The HVO (MVO), seen most clearly in the wings of the ${ }^{12} \mathrm{CO} J=2-1(\mathrm{SO} N, J=5,6-4,5$ and $\mathrm{SiO} J=5-4)$ emission lines, is compact with an angular size 1". $4(0$ "' 9$)$, and has a (projected) outflow velocity of $\sim 140 \mathrm{~km} \mathrm{~s}^{-1}$ $\left(\sim 30 \mathrm{~km} \mathrm{~s}^{-1}\right)$.

4. Because the sense of the velocity gradient, axis orientation, and scalar momentum of the HVO and SEO are roughly similar, the SEO is likely a result of the interaction of the HVO with the surrounding ambient cloud material.

5. The MVO emission produces prominent broad-wing emission in three of the four SO lines observed. The SO emission comes from a hot, shocked gas in the MVO at relatively high densities $\gtrsim 10^{7} \mathrm{~cm}^{-3}$ and temperatures $(\gtrsim 80 \mathrm{~K})$. The lowest feasible value of the density is $\sim 10^{6} \mathrm{~cm}^{-3}$, but then a high temperature $(>120 \mathrm{~K})$ is needed.

6. The pseudo-disk has a size of about 9600-11,100 au, and a thickness of $7400 \mathrm{au}$. A simple disk model with infall and rotation that fits its observed position-velocity structure gives a central stellar mass of $8.3-18.8 M_{\odot}$, and a disk mass of $0.64-0.85 M_{\odot}$. The disk mass is consistent, within uncertainties, with that derived from the millimeter-wave thermal dust emission $\left(1.7 M_{\odot}\right)$.

7. The wide-angle bullet spray shares a common axis with the pseudo-disk, and has an age comparable to that of MVO (few hundred years), suggesting that these three structures are intimately linked together.

8. A second-epoch imaging program with $H S T$ should be able to provide proper motions of nearby stars and help in distinguishing between three possible models (dynamical decay of a stellar cluster, chance encounter of a runaway star with a dense cloud, and close passage of two protostars) to explain the IRAS 05506 outflow source.

R.S.'s contribution to the research described here was carried out at the Jet Propulsion Laboratory (JPL), California Institute of Technology, under a contract with NASA. Financial support was provided by NASA, in part from HST/GO awards (nos. GO-09463.01-A and GO-09801.01-A) from the Space Telescope 
Science Institute (operated by the Association of Universities for Research in Astronomy, under NASA contract NAS5-26555). C.S.C. is supported by the Spanish MINECO through grants AYA2016-75066-C2-1-P and AYA2012-32032, and by the European Research Council through grant ERC-2013-SyG 610256. C.-F.L. acknowledges grants from the Ministry of Science and Technology of Taiwan (MoST 104-2119-M-001-015-MY3) and the Academia Sinica (Career Development Award). The National Radio Astronomy Observatory is a facility of the National Science Foundation operated under cooperative agreement by Associated Universities, Inc.

\section{ORCID iDs}

Raghvendra Sahai (i) https://orcid.org/0000-0002-6858-5063 Chin-Fei Lee (1D https://orcid.org/0000-0002-3024-5864 Carmen Sánchez Contreras (i) https://orcid.org/0000-00026341-592X

Mark R. Morris (ib https://orcid.org/0000-0002-6753-2066

\section{References}

Allen, D. A., \& Burton, M. G. 1993, Natur, 363, 54

Bally, J., Cunningham, N. J., Moeckel, N., et al. 2011, ApJ, 727, 113

Bally, J., Ginsburg, A., Arce, H., et al. 2017, ApJ, 837, 60

Bally, J., \& Zinnecker, H. 2005, AJ, 129, 2281

Bieging, J. H., \& Peters, W. L. 2011, ApJS, 196, 18

Chatterjee, S., \& Tan, J. C. 2012, ApJ, 754, 152

Goddi, C., Humphreys, E. M. L., Greenhill, L. J., Chandler, C. J., \& Matthews, L. D. 2011, ApJ, 728, 15

Goldsmith, P. F., Bergin, E. A., \& Lis, D. C. 1997, ApJ, 491, 615
Hartigan, P., Raymond, J., \& Hartmann, L. 1987, ApJ, 316, 323

Hogerheijde, M. R. 2001, ApJ, 553, 618

Lee, C.-F., Hirano, N., Zhang, Q., et al. 2014, ApJ, 786, 114

Lee, C.-F., Ho, P. T. P., Beuther, H., et al. 2006, ApJ, 639, 292

Lonsdale, C. J., Becklin, E. E., Lee, T. J., \& Stewart, J. M. 1982, AJ, 87, 1819

Luhman, K. L., Robberto, M., Tan, J. C., et al. 2017, ApJL, 838, L3

Lumsden, S. L., Hoare, M. G., Urquhart, J. S., et al. 2013, ApJS, 208, 11

Molinari, S., Schisano, E., Elia, D., et al. 2016, A\&A, 591, A149

Plambeck, R. L., \& Wright, M. C. H. 2016, ApJ, 833, 219

Plambeck, R. L., Wright, M. C. H., Friedel, D. N., et al. 2009, ApJL, 704, L25

Podio, L., Codella, C., Gueth, F., et al. 2015, A\&A, 581, A85

Qiu, K., Zhang, Q., Megeath, S. T., et al. 2008, ApJ, 685, 1005

Rodríguez, L. F., Poveda, A., Lizano, S., \& Allen, C. 2005, ApJL, 627, L65

Sahai, R., Claussen, M., Sánchez Contreras, C., Morris, M., \& Sarkar, G. 2008, ApJ, 680, 483

Sahai, R., te Lintel Hekkert, P., Morris, M., Zijlstra, A., \& Likkel, L. 1999, ApJL, 514, L115

Sánchez Contreras, C., \& Sahai, R. 2012, ApJS, 203, 16

Sánchez Contreras, C., Sahai, R., Gil de Paz, A., \& Goodrich, R. 2008, ApJS, 179,166

Sridharan, T. K., Beuther, H., Schilke, P., Menten, K. M., \& Wyrowski, F. 2002, ApJ, 566, 931

Tan, J. C. 2004, ApJL, 607, L47

Tenenbaum, E. D., Apponi, A. J., Ziurys, L. M., et al. 2006, ApJL, 649, L17

Tenenbaum, E. D., Dodd, J. L., Milam, S. N., Woolf, N. J., \& Ziurys, L. M. 2010, ApJS, 190, 348

van der Tak, F. F. S., Black, J. H., Schöier, F. L., Jansen, D. J., \& van Dishoeck, E. F. 2007, A\&A, 468, 627

Wilson, T. L. 1999, RPPh, 62, 143

Zapata, L. A., Rodríguez, L. F., Schmid-Burgk, J., et al. 2012, ApJL, 754, L17

Zapata, L. A., Schmid-Burgk, J., Ho, P. T. P., Rodríguez, L. F., \& Menten, K. M. 2009, ApJL, 704, L45

Zapata, L. A., Schmid-Burgk, J., Pérez-Goytia, N., et al. 2013, ApJL, 765, L29

Zhang, Y., Kwok, S., \& Dinh-V-Trung 2008, ApJ, 678, 328

Zinnecker, H., \& Yorke, H. W. 2007, ARA\&A, 45 